OPEN ACCESS

Edited by:

Georg Zoidl,

York University, Canada

Reviewed by:

Logan William Donaldson,

York University, Canada

Mauricio Antonio Retamal, Universidad del Desarrollo, Chile

Donglin Bai,

Western University, Canada

*Correspondence:

Tadas Kraujalis

tadas.kraujalis@/smuni.It

Specialty section: This article was submitted to Membrane Physiology and Membrane Biophysics,

a section of the journal

Frontiers in Physiology

Received: 19 December 2021

Accepted: 28 January 2022

Published: 21 February 2022

Citation:

Kraujalis T, Gudaitis $L$,

Kraujaliene L, Snipas $M$, Palacios-Prado N and Verselis VK (2022) The Amino Terminal Domain and Modulation of Connexin36 Gap Junction Channels by Intracellular

Magnesium lons.

Front. Physiol. 13:839223. doi: 10.3389/fphys.2022.839223

\section{The Amino Terminal Domain and Modulation of Connexin36 Gap Junction Channels by Intracellular Magnesium Ions}

\author{
Tadas Kraujalis ${ }^{1,2 *}$, Lukas Gudaitis ${ }^{1}$, Lina Kraujaliene ${ }^{1}$, Mindaugas Snipas ${ }^{1,3}$, \\ Nicolás Palacios-Prado ${ }^{4,5}$ and Vytas K. Verselis ${ }^{6}$
}

${ }^{1}$ Institute of Cardiology, Lithuanian University of Health Sciences, Kaunas, Lithuania, ${ }^{2}$ Department of Applied Informatics, Kaunas University of Technology, Kaunas, Lithuania, ${ }^{3}$ Department of Mathematical Modelling, Kaunas University of Technology, Kaunas, Lithuania, ${ }^{4}$ Department of Physiology, Pontificia Universidad Católica de Chile, Santiago, Chile, ${ }^{5}$ Centro Interdisciplinario de Neurociencias de Valparaíso, Universidad de Valparaiso, Valparaíso, Chile, ${ }^{6}$ Dominick P. Purpura Department of Neuroscience, Albert Einstein College of Medicine, New York, NY, United States

Electrical synapses between neurons in the mammalian CNS are predominantly formed of the connexin36 (Cx36) gap junction (GJ) channel protein. Unique among GJs formed of a number of other members of the Cx gene family, Cx36 GJs possess a high sensitivity to intracellular $\mathrm{Mg}^{2+}$ that can robustly act to modulate the strength of electrical synaptic transmission. Although a putative $\mathrm{Mg}^{2+}$ binding site was previously identified to reside in the aqueous pore in the first extracellular (E1) loop domain, the involvement of the $\mathrm{N}$-terminal (NT) domain in the atypical response of Cx36 GJs to $\mathrm{pH}$ was shown to depend on intracellular levels of $\mathrm{Mg}^{2+}$. In this study, we examined the impact of amino acid substitutions in the NT domain on $\mathrm{Mg}^{2+}$ modulation of $\mathrm{C} \times 36$ GJs, focusing on positions predicted to line the pore funnel, which constitutes the cytoplasmic entrance of the channel pore. We find that charge substitutions at the 8th, 13th, and 18th positions had pronounced effects on $\mathrm{Mg}^{2+}$ sensitivity, particularly at position 13 at which an A13K substitution completely abolished sensitivity to $\mathrm{Mg}^{2+}$. To assess potential mechanisms of $\mathrm{Mg}^{2+}$ action, we constructed and tested a series of mathematical models that took into account gating of the component hemichannels in a Cx36 GJ channel as well as $\mathrm{Mg}^{2+}$ binding to each hemichannel in open and/or closed states. Simultaneous model fitting of measurements of junctional conductance, $\mathrm{g}_{\mathrm{j}}$, and transjunctional $\mathrm{Mg}^{2+}$ fluxes using a fluorescent $\mathrm{Mg}^{2+}$ indicator suggested that the most viable mechanism for Cx36 regulation by $\mathrm{Mg}^{2+}$ entails the binding of $\mathrm{Mg}^{2+}$ to and subsequent stabilization of the closed state in each hemichannel. Reduced permeability to $\mathrm{Mg}^{2+}$ was also evident, particularly for the A13K substitution, but homology modeling of all chargesubstituted NT variants showed only a moderate correlation between a reduction in the negative electrostatic potential and a reduction in the permeability to $\mathrm{Mg}^{2+}$ ions. Given 
the reported role of the $\mathrm{E} 1$ domain in $\mathrm{Mg}^{2+}$ binding together with the impact of NT substitutions on gating and the apparent state-dependence of $\mathrm{Mg}^{2+}$ binding, this study suggests that the NT domain can be an integral part of $\mathrm{Mg}^{2+}$ modulation of Cx36 GJs likely through the coupling of conformational changes between NT and E1 domains.

Keywords: gap junction, connexin, intracellular $\mathrm{Mg}^{2+}$, ion permeability, electrophysiology

\section{INTRODUCTION}

Gap junction (G) channels, formed of connexin $(\mathrm{Cx})$ protein subunits, serve as intercellular communication pathways that enable the efficient propagation of electrical signals and the flux of small ions, amino acids, metabolites and peptides between cells (Niessen et al., 2000; Neijssen et al., 2005; Bedner et al., 2006). There are 20 Cxs expressed in rodents and 21 in humans and the $\mathrm{Cx} 36$ isoform is the main $\mathrm{Cx}$ expressed in neurons thereby constituting the bulk of the electrical synapses in the adult brain, as well as in retinal circuits (Connors and Long, 2004). Cx36 also has been identified in GJs between islet beta cells in rodent (Orci et al., 1973; Meda et al., 1979; Int Veld et al., 1986) and human pancreas (Serre-Beinier et al., 2008), where it plays a key role in regulating glucose homeostasis (Pérez-Armendariz, 2013).

Of particular relevance to electrical synapses is the extent to which the strength of electrical coupling can be modulated, which can substantially impact neuronal synchronization and the coordination of activity within neuronal networks (Bennett and Zukin, 2004; Bissiere et al., 2011). Forms of modulation include voltage dependence, expressed as a sensitivity to the transjunctional voltage $\left(\mathrm{V}_{\mathrm{j}}\right)$, the voltage difference between cells (Harris et al., 1981; Bukauskas and Verselis, 2004) and chemical factors, most notably intracellular $\mathrm{H}^{+}$(Trexler et al., 1999; Palacios-Prado et al., 2010) and intracellular divalent cations such as $\mathrm{Ca}^{2+}$ and $\mathrm{Mg}^{2+}$ (Noma and Tsuboi, 1987; Peracchia, 2004; Matsuda et al., 2010; Palacios-Prado et al., 2013). These forms of modulation are common among GJ channels, but sensitivities can differ substantially among the various $\mathrm{Cx}$ isoforms. For GJs formed of $\mathrm{Cx} 36$, there is a distinct aspect to their modulation by intracellular free $\mathrm{Mg}^{2+}$ (Palacios-Prado et al., 2013; Palacios-Prado et al., 2014). Using exogenous Cx expression in communication-deficient HeLa cells, junctional conductance, $\mathrm{g}_{\mathrm{j}}$, between cells expressing Cx36 was shown to increase robustly, several fold when the intracellular concentration of free $\mathrm{Mg}^{2+}$ ions, $\left[\mathrm{Mg}^{2+}\right]_{\mathrm{i}}$, was reduced from resting levels, which are typically $\sim 1 \mathrm{mM}$. This effect on $g_{j}$ has not been observed in other Cxs (Palacios-Prado et al., 2013). Conversely, when $\left[\mathrm{Mg}^{2+}\right]_{\mathrm{i}}$ was increased from resting levels, reductions in $g_{j}$ occurred. However, reductions in $\mathrm{g}_{j}$ upon elevating intracellular $\mathrm{Mg}^{2+}$ occur in GJs formed of other Cxs suggesting that Cx36 GJ channels exhibit a substantial shift in $\mathrm{Mg}^{2+}$ sensitivity such that changes in $\left[\mathrm{Mg}^{2+}\right]_{\mathrm{i}}$ at or about physiological levels can robustly impact junctional coupling. In rat brain slices, electrical synapses between neurons of the trigeminal mesencephalic nucleus, and between interneurons from the thalamic reticular nucleus, which are formed of $\mathrm{Cx} 36$, also were shown to exhibit sensitivity to reductions in $\left[\mathrm{Mg}^{2+}\right]_{\mathrm{i}}$ (Palacios-Prado et al., 2013; PalaciosPrado et al., 2014), indicative that this form of modulation occurs in native tissues and is a property that is intrinsic to the Cx36 isoform.

Changes in $\left[\mathrm{Mg}^{2+}\right]_{\mathrm{i}}$ can occur under a variety of physiological and pathological conditions. Since the majority of $\mathrm{Mg}^{2+}$ ions are bound to ATP, changes in cytoplasmic ATP levels can affect the levels of $\left[\mathrm{Mg}^{2+}\right]_{\mathrm{i}}$ (Luthi et al., 1999). Neuronal cytoplasmic ATP levels indeed have been shown to increase with glucose and lactate exposure (Ainscow et al., 2002) and during periods of reduced activity, such as sleep (Dworak et al., 2010). Conversely, cytoplasmic ATP levels were shown to decrease during periods of increased neuronal activity, such as those associated with wake periods and hyperactivity (Dworak et al., 2010). Pathologically, significant reductions in $\left[\mathrm{Mg}^{2+}\right]_{i}$ can occur following traumatic brain injury (Vink et al., 2009) and is a common feature in various neurological disorders, such as epilepsy, Parkinson's and Alzheimer's disease (Barbiroli et al., 1999; Andrasi et al., 2000; Fang et al., 2010). Elevation of $\left[\mathrm{Mg}^{2+}\right]_{\mathrm{i}}$ can occur in the brains of patients that have undergone ischemic events and that have been diagnosed with schizophrenia (Hinsberger et al., 1997) or mitochondrial disorders (Barbiroli et al., 1999). In the pancreas, deficiencies in $\mathrm{Mg}^{2+}$ levels have been associated with Type 2 diabetes mellitus (Barbagallo and Dominguez, 2007; Ramadass et al., 2015) and pancreatitis (Papazachariou et al., 2000). Thus, the robust sensitivity of $\mathrm{Cx} 36$ GJs to intracellular $\mathrm{Mg}^{2+}$ can have a large impact on neuronal cell function through modulation of electrical coupling, as well as the communication of signaling molecules and metabolites.

Using a chimeric approach that swapped domains between Cx36 and Cx43, the higher sensitivity of Cx36 GJs to $\left[\mathrm{Mg}^{2+}\right]_{i}$ was attributed, at least in part, to the first extracellular loop (E1) domain and in particular a charged residue, D47, predicted to be a pore-lining residue (Palacios-Prado et al., 2014). Subsequent studies, however, indirectly suggested that the N-terminal (NT) domain also may play a role in $\mathrm{Mg}^{2+}$ sensitivity of $\mathrm{Cx} 36$ GJs based on the observation that the effects of intracellular $\mathrm{pH}$ and $\left[\mathrm{Mg}^{2+}\right]_{\mathrm{i}}$ were interdependent (Rimkute et al., 2018). Acidification, which typically produces a robust reduction in $\mathrm{g}_{\mathrm{j}}$ for most GJs, uniquely produces an increase in $\mathrm{g}_{\mathrm{j}}$ for $\mathrm{Cx} 36$ GJs (González-Nieto et al., 2008). However, when $\left[\mathrm{Mg}^{2+}\right]_{\mathrm{i}}$ is reduced, the increase in $g_{j}$ no longer occurs suggesting, perhaps, that $\mathrm{pH}$ alters the binding of $\mathrm{Mg}^{2+}$ leading to differential effects of acidification on $g_{j}$ depending on the level of $\left[\mathrm{Mg}^{2+}\right]_{i}$. Molecularly, glutamate residues E8 and E12 in the NT domain were shown to play a role in this interdependence between $\mathrm{pH}$ and $\mathrm{Mg}^{2+}$, although only E8 appeared to impact the increase in $\mathrm{g}_{\mathbf{j}}$ observed upon reduction of $\left[\mathrm{Mg}^{2+}\right]_{\mathrm{i}}$ levels (Rimkute et al., 2018).

The aim of the present study was to more extensively examine the role of the NT domain in the modulation of Cx36 GJ channels by $\left[\mathrm{Mg}^{2+}\right]_{\mathrm{i}}$. We examined point mutations at 5 positions, E3, 
T5, E8, A13 and H18 in the NT domain of Cx36 that, based on homology modeling using the more recent $\mathrm{Cx} 46 / \mathrm{Cx} 50$ structure (Myers et al., 2018), are predicted to be exposed to the pore. In comparison to $\mathrm{Cx} 43$, which was used in the chimeric approach to examine the molecular determinants of $\mathrm{Mg}^{2+}$ regulation, two of the six positions differ in charge, E8 in Cx36 vs G8 in Cx43, and $\mathrm{A} 13$ in $\mathrm{Cx} 36$ vs $\mathrm{K} 13$ in $\mathrm{Cx} 43$. We find that charge substitutions at these two positions significantly altered the response of Cx36 to $\left[\mathrm{Mg}^{2+}\right]_{\mathrm{i}}$, as did charge substitution at H18. E8Q and $\mathrm{H} 18 \mathrm{~K}$ substitutions abolished the increase in $\mathrm{g}_{\mathrm{j}}$ upon exposure to low $\left[\mathrm{Mg}^{2+}\right]_{\mathrm{i}}$ and $\mathrm{A} 13 \mathrm{~K}$ completely abolished the effects of $\mathrm{Mg}^{2+}$ at all concentrations. Charge substitutions at the other positions had no effect. Combining electrophysiological recordings that compared the extent as well as the rate of the $\mathrm{Mg}^{2+}$ effect on $\mathrm{g}_{j}$ in all the mutants, along with measurements of $\mathrm{Mg}^{2+}$ permeability using a fluorescent indicator, we developed mathematical models to assess potential mechanisms of $\mathrm{Mg}^{2+}$ modulation of $\mathrm{Cx} 36 \mathrm{GJ}$ channels. These models were based on different kinetic schemes of $\mathrm{Mg}^{2+}$-binding and the putative effect of $\mathrm{Mg}^{2+}$ on the closing each of the component hemichannels in a Cx36 GJ channel. We found that $\mathrm{Mg}^{2+}$ most likely acts by binding to and stabilizing the closed conformation of a Cx36 hemichannel and that NT residues, which are at the cytoplasmic entrance of the pore, act electrostatically and allosterically to alter the permeability and/or binding of $\mathrm{Mg}^{2+}$ postulated to be deeper in the pore in the E1 domain.

\section{MATERIALS AND METHODS}

\section{Cells and Culture Conditions}

Electrophysiological measurements were performed in RIN cells (rat beta-cell insulinoma, ATCC CRL-2057) transfected with WT or mutant variants of mouse $\mathrm{Cx} 36$ fused with enhanced green fluorescent protein (EGFP) attached to the carboxy terminus. Cells were cultured in RPMI 1640 medium, with L-glutamine, supplemented with $10 \%$ fetal bovine serum, $1 \%$ streptomycin, and $1 \%$ penicillin. Mutants of Cx36 were generated using the QuikChange Multi Site-directed mutagenesis kit (Agilent). These mutants were subcloned into pEGFP-N1 vector (Clontech, United States) and transfected into RIN cells using Lipofectamine 2000 (Life technologies, United States). All experiments were performed with stable cell lines.

\section{In vitro Electrophysiological Measurements}

Electrophysiological recordings and fluorescence imaging were performed on cells grown on glass coverslips and transferred to an experimental chamber placed on the stage of an inverted microscope Olympus IX70 (Olympus, Japan) equipped with a fluorescence imaging system and a constant flow-through perfusion. Junctional conductance, $g_{j}$, was measured in cell pairs using the dual whole-cell patch clamp technique with EPC7 (HEKA) or EPC-8 (HEKA) patch clamp amplifiers. Voltage and current signals were digitized using an A/D converter (National Instrument) and acquired and analyzed using custommade software. Transjunctional voltages $\left(\mathrm{V}_{\mathrm{j}}\right)$ were generated by stepping the voltage in cell-1 while keeping the voltage in cell-2 constant. Junctional current $\left(\mathrm{I}_{\mathrm{j}}\right)$ was measured as the current change in cell-2. Patch pipette resistance was maintained below $3 \mathrm{M} \Omega$ in order to minimize the influence of series resistance on measurements of $g_{j}$. Recording pipettes were pulled from borosilicate glass capillary tubes with filaments using P-97 micropipette puller (Sutter Instrument Co., United States).

During recording, cells were perfused with an extracellular solution containing (in $\mathrm{mM}$ ): $140 \mathrm{NaCl}, 4 \mathrm{KCl}, 2 \mathrm{CaCl}_{2}, 1$ $\mathrm{MgCl}_{2}, 2 \mathrm{CsCl}, 1 \mathrm{BaCl}_{2}, 5 \mathrm{HEPES}, 5$ glucose, 2 pyruvate, $\mathrm{pH}$ 7.4. Patch pipettes were filled with a solution containing (in $\mathrm{mM}$ ): $130 \mathrm{CsCl}, 10 \mathrm{NaAsp}, 0.26 \mathrm{CaCl}_{2}, 5 \mathrm{HEPES}, 2 \mathrm{BAPTA}$, $1 \mathrm{MgCl}_{2}, \mathrm{pH}$ 7.3. To study the effect of $\left[\mathrm{Mg}^{2+}\right]_{\mathrm{i}}$ pipette solutions contained different concentrations of $\mathrm{MgCl}_{2}: 0.01$, 1 , or $5 \mathrm{mM}$. To chelate free $\mathrm{Mg}^{2+}$ ions in the cell we used $2 \mathrm{mM} \mathrm{K} \mathrm{K}_{2} \mathrm{ATP}$ in the pipette solution with $0.01 \mathrm{mM} \mathrm{Mg}^{2+}$. Maxchelator software was used to calculate free $\mathrm{Mg}^{2+}$ ionic concentrations. Solutions were adjusted for differences in osmolarity with the appropriate concentrations of $\mathrm{CsCl}$.

\section{Fluorescence Imaging Studies}

Fluorescence imaging was accomplished using an ORCA digital camera (Hamamatsu) and UltraVIEW (PerkinElmerLifeSciences, Boston, MA, United States) software. We used an excitation filter of 485, and emission filter of 530 to identify cell pairs expressing WT and mutant variants of Cx36. The same filters were used for fluorescence measurement of Mag-Fluo-4. For magnesium transfer studies, $\mathrm{a} \mathrm{Mg}^{2+}$ fluorescent indicator Mag-Fluo-4 (50 $\mu \mathrm{M})$ was introduced into cell-1 of a cell pair through a patch pipette with modified standard pipette solution without $\mathrm{MgCl}_{2}$. Cell-2 was patched with the pipette containing standard pipette solution and 5 or $10 \mathrm{mM}$ $\mathrm{MgCl}_{2}$. After breaking into cell-1, the fluorescence intensity $\left(\mathrm{FI}_{1}\right)$ of Mag-Flou-4 rose to a steady state value. The patch in cell-2 was then opened and the changes in $\mathrm{FI}_{1}$ in cell-1 and $\mathrm{g}_{j}$ were measured simultaneously. To measure $\mathrm{g}_{j}$, repeated, small amplitude voltage ramps $( \pm 10 \mathrm{mV})$ were applied.

\section{Homology Structure Modeling}

Structural homology models of Cx36 and its mutants were built using as a template the structure of a $\mathrm{Cx} 46 / \mathrm{Cx} 50$ GJ channel (6MHQ) obtained by cryo-EM (Myers et al., 2018). Homology modeling was carried out using MODELLER. The sequence of Cx36 was aligned with that of Cx46 using MODELLER software and showed a sequence identity of $>53 \%$. The best model was selected according to the discrete optimized protein energy score. Input files for building the Cx36 homology model using MODELLER software are provided in Supplementary Material.

\section{Simulation of the Electrostatic Potential}

The electrostatic potentials of all the atoms in the protein were estimated using Adaptive Poisson-Boltzman solver (APBS). To convert PDB files into PQR as input to APBS, we used PDB2PQR server with a PARSE forcefield (Dolinsky et al., 2004). PROPKA was used to assign protonation states at $\mathrm{pH}$ 7.3. Dielectric constants were set to 2.0 for the interior (protein) and 80.0 for the exterior (solvent) regions. To calculate electrostatic potentials at 
a particular location, data from the DX file of the corresponding model were extracted and analyzed using custom-made software written in C\# programming language. The visualization was done using Visual Molecular Dynamics and UCSF Chimera software.

\section{Minimization and Equilibration}

Introducing mutations adds features that may not be structurally accurate. To improve the accuracy of the system structure, we performed minimization and equilibration using GROMACS simulation software. The protein was inserted in a lipid bilayer membrane environment using the CHARMM-GUI membrane builder (Wu et al., 2014; Lee et al., 2018). The inner and outer leaflets of the membrane consisted of mixed lipids: phospholipid (PA, PC, PE, PI, and PS) (Locke and Harris, 2009), sphingolipids (SSM and LSM), and sterols (Cholesterol) (Cascio, 2005). The assembled systems were solvated with a sufficient number of TIP3P water molecules. To neutralize the systems, $0.15 \mathrm{M} \mathrm{NaCl}$ solution was added. Temperature and pressure were maintained at $303.15 \mathrm{~K}\left(30^{\circ} \mathrm{C}\right)$ and 1 bar, respectively. The system energy was minimized using the steepest descent algorithm until the maximum force went below $1000 \mathrm{~kJ} / \mathrm{mol} / \mathrm{nm}$. Each system was equilibrated using input files generated by CHARMM-GUI. To ensure a valid equilibration, each system was verified through investigating the root-mean-square deviation.

\section{Mathematical Models of $\mathbf{M g}^{2+}-$ Induced GJ Channel Gating and Ion Permeability Magnesium Binding and Channel Closure}

We developed a series of different mathematical models to examine possible mechanisms of $\mathrm{Mg}^{2+}$ action on GJ channels formed of $\mathrm{Cx} 36$. All the models were based on the assumption, that $\mathrm{Mg}^{2+}$ can bind to each of the apposing hemichannels and that each hemichannel can be described by a linear 3-state reaction scheme of the following form:

$$
O \underset{k_{2}}{\stackrel{k_{1}}{\rightleftarrows}} X \underset{k_{4}}{\stackrel{k_{3}}{\rightleftarrows}} C .
$$

Here, $O$ and $C$ denote open and closed states of a hemichannel, respectively. $X$ denotes an intermediate state, which, could be either open or closed (for simplicity, we did not consider any intermediate values of conductance for this state of the hemichannel). In addition, we presumed that $O \rightarrow X$ and $X \rightarrow C$ transitions could be either $\mathrm{Mg}$-independent or could reflect the binding of $\mathrm{Mg}^{2+}$ ions according to the law of mass action. This gives us eight different models to consider, depending on the assumptions about the intermediate state $X$, as well as $O \rightarrow X$ and $X \rightarrow C$ transitions (see Table $\mathbf{1}$ ).

As is noted in Table 1, we discarded two models which did not contain any $\mathrm{Mg}^{2+}$ ion binding (denoted by ${ }^{*}$ ), and another model (denoted by ${ }^{\times}$), which exhibits a redundant $\mathrm{Mg}$-independent transition between two open states. This leaves us with five basic models, each describing somewhat different mechanistic scenarios. For example, in Model $1, \mathrm{a} \mathrm{Mg}^{2+}$ binding event occurs in a hemichannel in its open conformation, which remains open until a subsequent binding event occurs resulting in hemichannel

\begin{tabular}{|c|c|c|c|}
\hline Model & $O \rightarrow X$ & $x$ & $X \rightarrow C$ \\
\hline Model 1 & $\mathrm{Mg}^{2+}$ binding & open & $\mathrm{Mg}^{2+}$ binding \\
\hline Model 2 & $\mathrm{Mg}^{2+}$ binding & open & Mg-independent \\
\hline Model 3 & $\mathrm{Mg}^{2+}$ binding & closed & $\mathrm{Mg}^{2+}$ binding \\
\hline Model 4 & $\mathrm{Mg}^{2+}$ binding & closed & Mg-independent \\
\hline Discarded $^{\times}$ & Mg-independent & open & $\mathrm{Mg}^{2+}$ binding \\
\hline Discarded * & Mg-independent & open & Mg-independent \\
\hline Model 5 & Mg-independent & closed & $M^{2+}$ binding \\
\hline Discarded * & Mg-independent & closed & Mg-independent \\
\hline
\end{tabular}

TABLE 1 | Different variants of a basic 3-state linear kinetic scheme $O \leftrightarrow X \leftrightarrow C$ to describe $\mathrm{Mg}^{2+}$-mediated closure of $\mathrm{C} \times 36$ hemichannels.

*-Does not contain Mg-dependent transitions.

X-Redundant Mg-independent transition between two open states.

closure, whereas in Model 5, a $\mathrm{Mg}^{2+}$ binding event occurs in a hemichannel in its closed conformation, with closure achieved through a $\mathrm{Mg}^{2+}$-independent gating event. For each of the five basic models, in addition to $\mathrm{Mg}^{2+}$ binding according to the law of mass action, we considered the Hill equation to describe $\mathrm{Mg}^{2+}$ binding, which can be interpreted as simultaneous binding of $n$ $\mathrm{Mg}^{2+}$ ions, or more generally, expresses cooperative $(n>1)$, competitive $(n<1)$ or independent $(n=1)$ binding (Yifrach, 2004). This consideration extended our analyses to a total of 10 models, with the number of free parameters varying between five and eight. Model testing was accomplished by fitting a collection of experimental data on WT and variant Cx36 GJs. The numerical model fitting experiments did not permit distinction among more complex models due to high parameter uncertainty and overfitting.

\section{Intracellular Concentration of $\mathrm{Mg}^{2+}$ lons}

The flux of $\mathrm{Mg}^{2+}$ ions through GJ channels was simulated in the same way for all models. Because $g_{j}$ values of cell pairs expressing WT and mutant variants of $\mathrm{Cx} 36$ were typically low, $\mathrm{Mg}^{2+}$ flux was relatively low and the $\mathrm{Mg}^{2+}$ ion concentration of the donor cell could be presumed to remain constant and equal to the value in the patch pipette throughout the duration of the experiments. For modeling, we presumed that the rate of diffusion of $\mathrm{Mg}^{2+}$ ions was proportional to the concentration difference between the cells as described by the Fick's law, which can be described by the following system of ordinary differential equations (ODEs):

$$
\left\{\begin{array}{l}
\frac{d\left[\mathrm{Mg}^{2+}\right]_{1}}{d t}=-P \cdot g_{\mathrm{j}} \cdot\left(\left[\mathrm{Mg}^{2+}\right]_{2}-\left[\mathrm{Mg}^{2+}\right]_{1}\right) \\
\frac{d\left[\mathrm{Mg}^{2+}\right]_{2}}{d t}=-P \cdot g_{\mathrm{j}} \cdot\left(\left[\mathrm{Mg}^{2+}\right]_{1}-\left[\mathrm{Mg}^{2+}\right]_{2}\right)
\end{array}\right.
$$

Here, $i(i=1,2)$ denotes the hemichannel in each of two apposing cells, cell- 1 and cell- 2 . The constant $P$ quantifies the permeability of a GJ channel to $\mathrm{Mg}^{2+}$ ions and $g_{\mathrm{j}}\left(0 \leq g_{\mathrm{j}} \leq 1\right)$ is normalized junctional conductance, which can also be interpreted as the proportion of open channels between two cells. Because the inspected RIN cells were of similar sizes we made the simplifying assumption that both cells of a pair were of the same volume. Any differences in cell volumes should average out in different experiments and should not distort modeling results to any significant degree. In addition, to simulate experiments with highly asymmetric concentrations of $\mathrm{Mg}^{2+}$ in the pipette 
solutions, we subtracted a component $P_{\text {leak }} \cdot\left[\mathrm{Mg}^{2+}\right]_{\mathrm{i}}$ to account for the leak of $\mathrm{Mg}^{2+}$ into the patch pipette in the recipient cell. The magnitude of this leak current parameter $P_{\text {leak }}$ was estimated as described in (Palacios-Prado et al., 2013).

\section{Model Fitting and Parameter Estimation}

Parameter estimation was performed using the MATLAB global optimization toolbox. To estimate model parameters, the simulated changes in $\mathrm{g}_{j}$ and intracellular $\mathrm{Mg}^{2+}$ over time were fit simultaneously to averaged electrophysiological and fluorescent imaging data for WT Cx36 and each variant. That is, we assumed that WT Cx36 and each of the variants can exhibit a distinct set of model parameters, which describes its $\mathrm{Mg}^{2+}$ binding affinity, permeability, etc. To solve a system of ODEs we applied the Euler method; the integration step was chosen to be sufficiently small (in 0.001-0.00001 range) to ensure the stability of the calculations. To avoid parameter redundancy and overfitting, we presumed fluorescence intensity of the $\mathrm{Mg}^{2+}$ indicator was proportional to the intracellular $\mathrm{Mg}^{2+}$ concentration and the constant of proportionality should be reflected in parameter $P$, when simulated $\mathrm{Mg}^{2+}$ concentration curves were fitted directly to measured changes in fluorescence intensity over time.

We applied the method of least squares for model fitting. That is, global optimization was performed to obtain model parameters which minimized the sum of squared errors (SSE):

$$
S S E=\sum_{i=1}^{n}\left(y_{i}-\hat{y}_{\mathrm{i}}\right)^{2} .
$$

Here $y_{i}$ denotes data points obtained from the experiments while $\hat{y}_{\mathrm{i}}$ denotes the respective values predicted by a model; $n$ is total number of experimentally observed values.

In theory, inclusion of an additional parameter into a model can only result in a lower SSE during the model fitting, even if the addition carries little or no relevance. Thus, to avoid such overfitting we used the Akaike Information Criterion (AIC). For least squares model fitting problems, AIC can be estimated from the following equation:

$$
A I C=2(k+1)+n \cdot \ln (S S E)-n \cdot \ln (n) .
$$

Here $k$ denotes the number of model parameters.

The value of AIC for a given model is useful only in comparison to AIC values of other models. That is, a model with the minimum value of AIC relative to all the others is considered the best. An increase in the number of model parameters raises the AIC value. Thus, such an addition is only justified if it significantly reduces the value of SSE. In this way, the use of AIC for model selection gives a preference for a more parsimonious model. In addition, it allows for a direct comparison among models which have different numbers of parameters.

If two models are applied for the same experimental dataset, they can only differ in the values of the delta AIC ( $\triangle \mathrm{AIC})$, which is defined as follows:

$$
\triangle A I C=2 k+n \cdot \ln (S S E) .
$$

As a rule of thumb, two candidate models are considered significantly different in fitting ability if the difference in their $\triangle \mathrm{AIC}$ values exceeds 2 (Symonds and Moussalli, 2011).

To compare different models we used the Akaike weights $\left(w_{\mathrm{i}}\right)$, which are defined as follows:

$$
w_{i}=\frac{\exp \left(-\Delta A I C_{i} / 2\right)}{\sum_{j=1}^{m} \exp \left(-\Delta A I C_{i} / 2\right)} .
$$

Here $\triangle A I C_{i}$ denotes the value of $\triangle \mathrm{AIC}$ of a respective model and $\mathrm{m}$ is the number of candidate models. The Akaike weight can be interpreted as the probability that the respective candidate model is the best among all the candidate models.

\section{Data Analysis}

The analysis and statistics were performed using RStudio and custom-made software written in C\# or Visual Basic for Applications languages. Statistical analyses were performed using an unpaired Student t-test. Differences were considered statistically significant at $p<0.05$.

\section{RESULTS}

\section{Charge Substitutions in the NT Domain Can Significantly Alter Regulation of Cx36 GJs by $\mathbf{M g}^{2+}$}

The predicted structure of the Cx36 GJ channel based on homology modeling using the $\mathrm{Cx} 46 / \mathrm{Cx} 50$ cryo-EM structure is shown in Figure 1A. Given that the molecular basis for the unique $\mathrm{Mg}^{2+}$ regulation of $\mathrm{Cx} 36$ was investigated by swapping domains with $\mathrm{Cx} 43$, Figure $1 \mathrm{~B}$ includes the sequence alignment for the NT domain of $\mathrm{Cx} 43$ along with $\mathrm{Cx} 36$ and $\mathrm{Cx} 46$. Positions $3,5,8,13$, and 18 in the NT domain of Cx36 are predicted to be exposed to the pore with A13 and H18 located at the wide cytoplasmic entrance of the pore vestibule. We individually mutated each of these residues and examined the effects on regulation by the intracellular $\mathrm{Mg}^{2+}$ ion concentration. To assess regulation by intracellular $\mathrm{Mg}^{2+}$, we measured $\mathrm{g}_{j}$ in pairs of cells expressing WT or variants of Cx36 with three different concentrations of $\mathrm{Mg}^{2+}$ in the patch pipettes, $0.01,1.0$, and $5.0 \mathrm{mM}$. At each $\mathrm{Mg}^{2+}$ concentration we measured the change in $g_{j}$ over time, noting the initial value upon establishing a dual whole cell recording, $\left(g_{j}\right.$, init $)$ and the final steady-state value $\left(g_{j, s s}\right)$. An index of the effect of $\mathrm{Mg}^{2+}$ was given by the ratio $\mathrm{g}_{\mathrm{j}, \mathrm{ss}} / \mathrm{g}_{\mathrm{j}, \text { init }}$.

Results for each of the substituted variants at positions, 3, 5, 8,13 , and 18, are summarized in Figure 2. Plots are shown for mean values of $g_{j}$,init (Figure $\mathbf{2 A}$ ) and $\mathrm{g}_{\mathrm{j}, \mathrm{ss}} / \mathrm{g}_{\mathrm{j}, \text { init }}$ (Figure $\mathbf{2 B}$ ). Considering the two negatively charged putative pore-lining Glu residues at positions, 3 and 8, E3 is conserved in charge compared to $\mathrm{Cx} 43$, whereas $\mathrm{E} 8$ is a neutral Gly residue. We neutralized each of these positions by replacing Glu with Gln. E3Q GJ channels behaved similar to WT Cx36 GJ channels, except for small, albeit significant reduction in $\mathrm{g}_{\mathrm{j}, \mathrm{ss}} / \mathrm{g}_{\mathrm{j}, \text { init }}$ that was accompanied by a lower mean value of $g_{j}$, init. The E8Q mutation, however, essentially abolished the increase in $\mathrm{g}_{\mathrm{j}}$ at low $\left[\mathrm{Mg}^{2+}\right]_{\mathrm{i}}$, 
$\mathbf{A}$

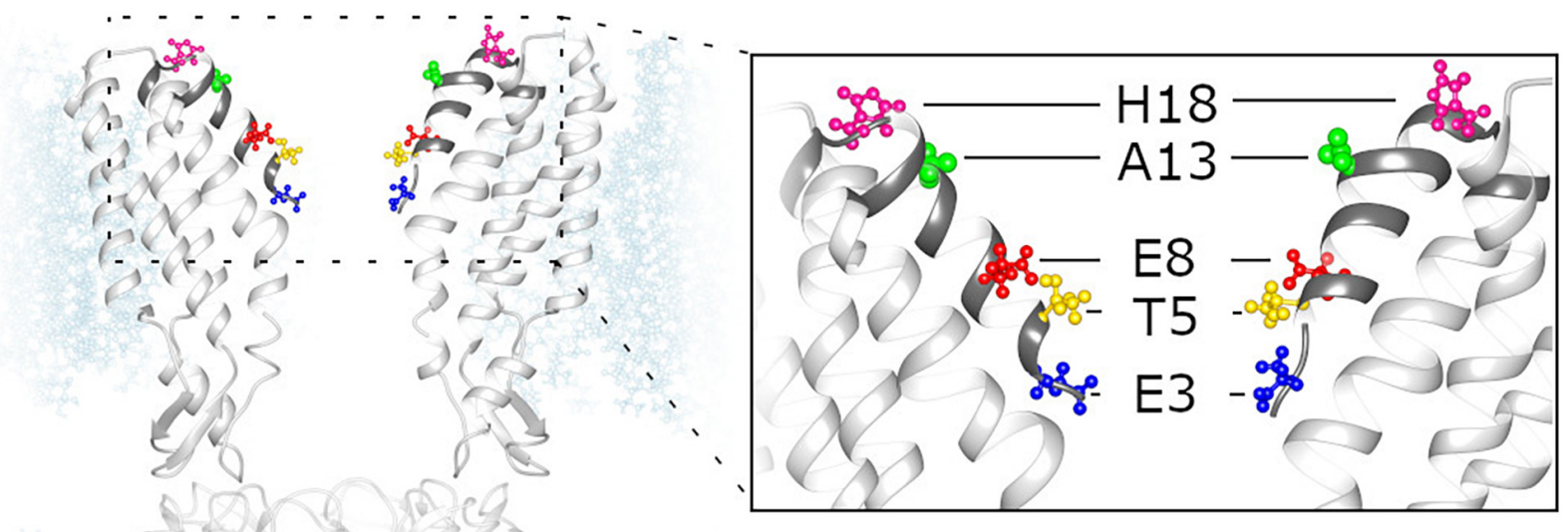

B

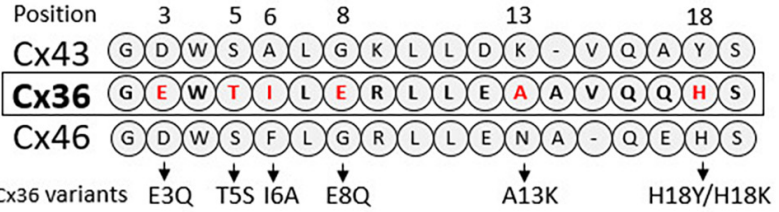

FIGURE 1 | Homology model of Cx36 GJ channel structure. (A) Cx36 GJ channel based on crystal structure of Cx46 (6MHQ) following energy minimization and equilibration. (Inset) The segment of GJ channel corresponding to NT domain (dark gray). The color-coded residues of Cx36 were replaced by the residues presented in panel (B). (B) Sequence alignment of Cx43, Cx36 and Cx46 NT domains.

but retained sensitivity to high $\left[\mathrm{Mg}^{2+}\right]_{\mathrm{i}}$. The mean value for $\mathrm{g}_{\mathrm{j}}$, init was not significantly different from WT Cx36.

Position 13, which is an Ala residue in $\mathrm{Cx} 36$, has a corresponding positively charged Lys in Cx43. Thus, we replaced A13 with Lys. The A13K mutation resulted in a complete loss of the response to all applied concentrations of $\mathrm{Mg}^{2+}$ (Figure 2B). Correspondingly, the $\mathrm{A} 13 \mathrm{~K}$ mutation showed a robust increase in the mean value of $\mathrm{g}_{\mathrm{j}}$, init. Moving further out toward the cytoplasmic vestibule, we replaced $\mathrm{H} 18$ with Lys which also resulted in significantly reduced sensitivity to intracellular $\mathrm{Mg}^{2+}$. Like A13K, H18K also exhibited a significantly higher mean value of $g_{j}$,init compared with WT Cx36 (Figure 2A). Neutralto-neutral substitutions at positions 5, and 18, T5S and H18Y, did not show any significant differences in sensitivity to intracellular $\mathrm{Mg}^{2+}$ compared to WT Cx36. An I6A mutation resulted in lost channel function (data not shown) consistent with the roles of non-pore-lining residues in the maintenance of NT structure (Purnick et al., 2000a). Thus, overall, the results suggest that alterations in charge in NT residues can significantly affect sensitivity of Cx36 GJs to intracellular $\mathrm{Mg}^{2+}$.

\section{Evaluation of $\mathrm{Mg}^{2+}$ Permeability and Changes in $\mathrm{g}_{\mathrm{j}}$ in WT and NT Mutant Cx36 GJ Channels}

Given that the NT domains constitute the cytoplasmic vestibules of a GJ channel pore, we wanted to evaluate whether the NT substitutions, particularly those that affected regulation by $\mathrm{Mg}^{2+}$, affected $\mathrm{Mg}^{2+}$ permeation. To assess permeability of $\mathrm{Mg}^{2+}$ through WT, E3Q, E8Q, A13K and H18K Cx36 GJ channels, we used a cell-impermeant fluorescent $\mathrm{Mg}^{2+}$ indicator Mag-Fluo4 (MF4). Experiments were performed using the same protocol as described in Palacios-Prado et al. (2013) in which we first obtained a whole-cell recording with a Mag-Fluo-4-containing pipette on one cell (cell-1) and then introduced $\mathrm{Mg}^{2+}$ in the other cell (cell-2) by establishing a second whole-cell recording with a $\mathrm{Mg}^{2+}$-containing pipette. This allowed simultaneous measurement of $g_{j}$ and changes in Mag-Fluo-4 fluorescence arising from the flux of $\mathrm{Mg}^{2+}$ through GJ channels. The effects of $\mathrm{Mg}^{2+}$ occurred over a time course of a few minutes during which time we saw no measurable changes in plaque size.

We used two concentrations of $\mathrm{Mg}^{2+}$ in the patch pipette for cell-2, 5 and $10 \mathrm{mM}$. The mean initial $g_{j}$ values (Figure 3A) were typically low in cell pairs expressing WT Cx36, E3Q, and E8Q, whereas they were higher in those expressing $A 13 \mathrm{~K}$ and $\mathrm{H} 18 \mathrm{~K}$, consistent with results from experiments summarized in Figure 2 under symmetric $\mathrm{Mg}^{2+}$ conditions. Changes in $\mathrm{g}_{\mathrm{j}}$ and Mag-Fluo-4 fluorescence at the end of a 5 min interval are shown in Figures 3B,C, respectively. To permit the assessment of mean values, for each cell pair, the data were normalized to the initial values of $g_{j}, g_{j}$, final $/ g_{j}$,init, and the initial fluorescence in cell-1. Changes in $g_{j}$ and fluorescence were followed after establishment of a whole-cell patch recording in cell-2. Again, consistent with results in Figure 2, all the variants, except for A13K, exhibited 


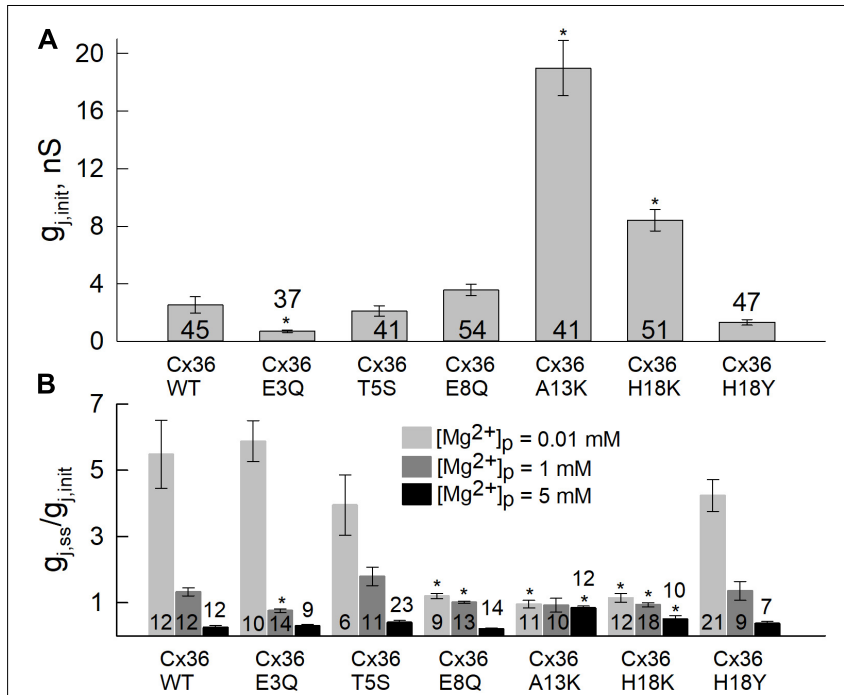

FIGURE 2 | Differences in sensitivity to $\left[\mathrm{Mg}^{2+}\right]_{\mathrm{p}}$ between Cx36 WT and NT variants. Experiments were performed in pairs of RIN cells expressing $\mathrm{C} \times 36$ WT and NT variants, tagged with EGFP. (A) Initial junctional conductance $\left(g_{j, \text { init }}\right)$ was registered immediately after the patch was opened. (B) Average $g_{j}$ value of $\mathrm{Cx36}$ and mutants at the steady-state level, normalized to initial

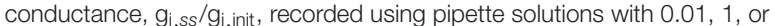
$5 \mathrm{mM}\left[\mathrm{Mg}^{2+}\right]_{p}$. Statistically significant $(p<0.05)$ differences of each mutant compared to WT Cx36 are indicated by asterisks. Data were obtained by applying repeated small voltage ramps from -10 to $10 \mathrm{mV}$. Error bars correspond to standard errors, and total numbers of experiments are indicated within or above the columns.

reductions in $\mathrm{g}_{\mathrm{j}}$ when high $\mathrm{Mg}^{2+}$ was introduced into the cytoplasm of cell-2. The magnitudes of the changes in $\mathrm{g}_{\mathrm{j}, \text { final }} / \mathrm{g}_{\mathrm{j}, \text { init }}$ for E3Q and E8Q GJs were similar to WT Cx36, although both variants, especially E8Q, exhibited somewhat slower kinetics compared to WT (see Figure 4). Also, gj decreased significantly more when the pipette contained $10 \mathrm{mM} \mathrm{Mg}^{2+}$ compared to $5 \mathrm{mM} \mathrm{Mg}^{2+}$. H18K, although exhibiting sensitivity to high $\mathrm{Mg}^{2+}$, showed no difference between 5 and $10 \mathrm{mM} \mathrm{Mg}^{2+}$. For these same cell pairs, the measured Mag-Fluo-4 fluorescence showed similar changes for E3Q and E8Q compared to WT. $\mathrm{A} 13 \mathrm{~K}$ and $\mathrm{H} 18 \mathrm{~K}$ GJs showed somewhat larger increases in fluorescence, $\mathrm{FI}_{1}$, but $\mathrm{g}_{\mathrm{j}}$,init in these cell pairs was significantly higher suggesting a substantially reduced permeability to $\mathrm{Mg}^{2+}$ when normalized to conductance. In the next section, we take all these data into account and apply mathematical models to gain insights into mechanisms of $\mathrm{Mg}^{2+}$ action.

\section{Fitting Mathematical Models to Electrophysiological and Fluorescent Imaging Data}

For model fitting, we used the 10 different 3 -state models detailed in Methods. These models were fit to the electrophysiological and fluorescence imaging data presented in Figures 3A,C using global optimization. For independent validation, we examined how well the obtained parameters could explain the reductions in $\mathrm{g}_{j}$ for WT and mutant variants of Cx36 recorded from separate data sets that measured the changes in $g_{j}$ over time under different conditions, namely both pipettes containing high $\mathrm{Mg}^{2+}$ (symmetric $5 \mathrm{mM}\left[\mathrm{Mg}^{2+}\right]_{\mathrm{p}}$ ). Table 2 presents the values of summary $\triangle \mathrm{AIC}$ and Akaike weights for model fitting data, as well as SSEs for independent validation $(\triangle \mathrm{AIC}$ and SSE values grouped by $\mathrm{Cx} 36$ variants are presented in Supplementary Material).

Data in Table 2 shows that that Model 5 with the Hill equation for $\mathrm{Mg}^{2+}$ binding provided the best fit. The difference between $\triangle \mathrm{AICs}$ of the best and second best model (Model 4 with the Hill equation) exceeds 2 , which is considered significant. The ratio of the respective Akaike weights $(\sim 1.91)$ indicates that Model 5 with the Hill equation has approximately a two times higher probability of being better than Model 4 with the Hill equation and is the best among all the considered models. In addition, Model 5 with the Hill equation provided the lowest SSE for independent validation data, which further strengthens our confidence that it is the most suitable candidate model.

The kinetic scheme of this model (Model 5, Hill) is presented below:

$$
\begin{aligned}
& \mathrm{O} \underset{k_{2}}{\stackrel{k_{1}}{\rightleftarrows} C} \\
& C+n \cdot \mathrm{Mg}^{2+} \underset{k_{4}}{\stackrel{k_{3}}{\rightleftarrows} \mathrm{C}-\mathrm{Mg}^{2+}}
\end{aligned}
$$

The first transition of this model is Mg-independent, which can be interpreted as stochastic gating of a hemichannel that is $\mathrm{V}_{\mathrm{j}}$-dependent. The second transition can be interpreted as the binding of $\mathrm{Mg}^{2+}$ ion(s) to the closed state of a hemichannel and subsequent stabilization of this closed state. The system of ODEs, describing $\mathrm{Mg}^{2+}$-binding and closure of a single hemichannel is presented below:

$$
\left\{\begin{array}{c}
\frac{d[O]_{i}}{d t}=-k_{1} \cdot[O]_{i}+k_{2} \cdot[C]_{i} \\
\frac{d[C]_{i}}{d t}=k_{1} \cdot[O]_{i}-\left(k_{2}+k_{3} \cdot\left[\mathrm{Mg}^{2+}\right]_{i}^{n}\right) \cdot[C]_{i}+k_{4} \cdot\left[\mathrm{C}-\mathrm{Mg}^{2+}\right]_{i} \\
\frac{d\left[\mathrm{C}-\mathrm{Mg}^{2+}\right]_{i}}{d t}=k_{3} \cdot[\mathrm{C}]_{i} \cdot\left[\mathrm{Mg}^{2+}\right]_{i}^{n}-k_{4} \cdot\left[\mathrm{C}-\mathrm{Mg}^{2+}\right]_{i}
\end{array}\right.
$$

Here, $i(i=1,2)$ denotes the hemichannel in each of two apposing cells, cell-1 or cell- 2 and $n$ is the Hill coefficient for the binding of $\mathrm{Mg}^{2+}$ ions. State variables $[\mathrm{O}]_{i},[\mathrm{C}]_{i}$ and $\left[\mathrm{C}-\mathrm{Mg}^{2+}\right]_{i}$ each denotes the probability that a hemichannel resides in an open, closed or $\mathrm{Mg}^{2+}$-stabilized closed state, respectively. These variables must be in the range $[0 ; 1]$ and satisfy the following conservation rule:

$$
[O]_{i}+[C]_{i}+\left[\mathrm{C}-\mathrm{Mg}^{2+}\right]_{i}=1 .
$$

Based on the assumption that each apposing hemichannel can close the GJ channel pore, $g_{j}$ can be expressed as $g_{j}=[O]_{1} \cdot[O]_{2}$. The estimated parameters of this model for WT and mutated Cx36 channels are presented in Table 3. The kinetic schemes and estimated parameters obtained from other considered models are presented in Supplementary Material.

The estimated values of model parameters suggest that the observed slower changes in $g_{j}$ for E3Q and E8Q as compared 

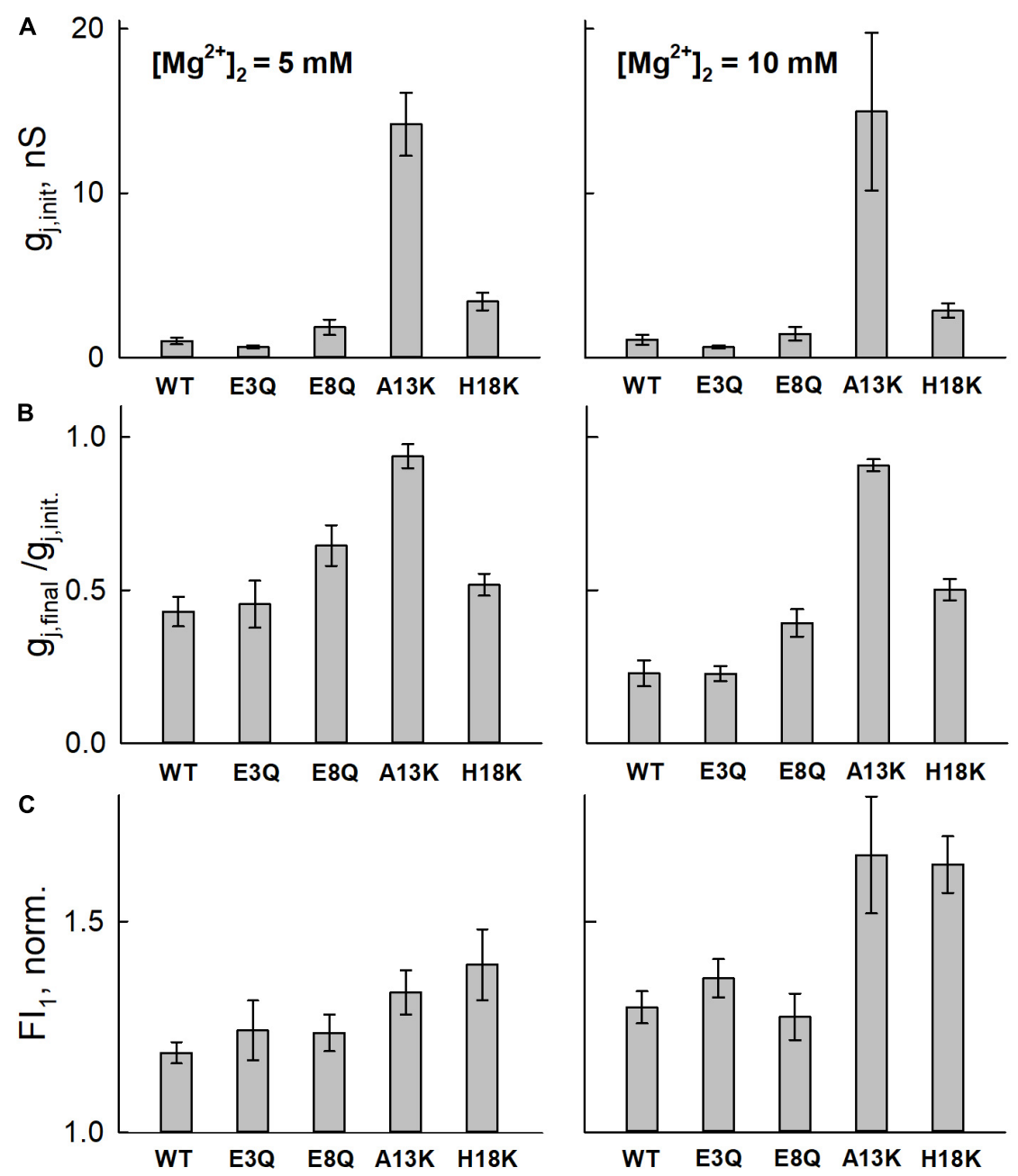

FIGURE 3 | Averaged data from simultaneous electrophysiological and fluorescence recordings in RIN cells expressing WT or mutated Cx36 GJ channels. (A) Bar graphs of initial $g_{j}$ values $\left(g_{j}\right.$, init), measured upon establishing a whole-cell patch recording in the second cell of a pair (cell-2) with a pipette containing high intracellular $\mathrm{Mg}^{2+}$. Thus, $\mathrm{g}_{\mathrm{j}}$,init is a measure of $\mathrm{g}_{\mathrm{j}}$ prior to any appreciable changes in intracellular $\mathrm{Mg}^{2+}$. (B) Mean $\mathrm{g}_{\mathrm{j}}\left(\mathrm{g}_{\mathrm{j}}\right.$, final) assessed at the end of a 5 minute time interval normalized to initial value $\left(g_{j}\right.$, init) assessed at the time a whole cell recording was established in cell-2. (C) Measurement of the change in Mag-Fluor-4 fluorescence in the same recipient cells (cell-1; $\mathrm{Fl}_{1}$ ) at the end of the $5 \mathrm{~min}$ time interval. $\mathrm{Fl}_{1}$ was normalized to the initial value approximating the resting $\mathrm{Mg}{ }^{2+}$ level. All experiments were performed with pipette-2 containing $5 \mathrm{mM}$ (first column) or $10 \mathrm{mM}$ (second column) Mg ${ }^{2+}$. Values represent the mean and standard error; $n=6$ for $5 \mathrm{mM} \mathrm{Mg}^{2+}$ and $n=5$ for $10 \mathrm{mM} \mathrm{Mg}^{2+}$.

to WT GJ channels mostly result from a decreased dissociation of $\mathrm{Mg}^{2+}$ ions from the putative binding site. As for WT $\mathrm{Cx} 36$, the estimated value of the Hill coefficient for both E3Q and E8Q variants was close to 1 , which indicates the absence of either cooperative or competitive binding of $\mathrm{Mg}^{2+}$ ions. The values of parameter $P$ indicate that E3Q exhibited an increased permeability to $\mathrm{Mg}^{2+}$ ions whereas E8Q exhibited a decrease compared to WT. The estimated parameters for A13K reflect two main distinguishing features. First, A13K was the only variant that resulted in a substantially reduced $\mathrm{Mg}^{2+}$ binding rate, as indicated by a very low value of $k_{3}$, or more precisely, the reduced ratio $k_{3} / k_{4}$, compared to WT and the other variants. In addition, the estimated value of $P$ indicates that $\mathrm{A} 13 \mathrm{~K}$ exhibits a more than 10 -fold reduction in permeability to $\mathrm{Mg}^{2+}$ ions. $\mathrm{H} 18 \mathrm{~K}$ also exhibited reduced permeability to
$\mathrm{Mg}^{2+}$, but similar in magnitude to E8Q. Compared to WT Cx36, however, H18K also showed an increase in stochastic gating activity at $\mathrm{V}_{\mathrm{j}}=0 \mathrm{mV}$ (see larger $k_{1}$ value) as well as a much lower value of the Hill coefficient. The latter may indicate a very low cooperativity for $\mathrm{Mg}^{2+}$ ion binding and could explain saturation of the $\mathrm{Mg}^{2+}$ effects on $\mathrm{g}_{j}$ sensitivity at $5 \mathrm{mM}$. In addition, it would result in a lower sensitivity to high $\mathrm{Mg}^{2+}$ concentrations.

The solid lines in Figure 4 represent the fits to the data and show that this model can account for the changes in $g_{j}$ and MagFluo-4 fluorescence (Figures 4A,B). Using the same parameters obtained from these fits, simulated responses (solid lines) fit an independent data set quite well both in terms of the magnitude and the kinetic changes in $g_{j}$ under conditions in which both patch pipettes containing $5 \mathrm{mM} \mathrm{Mg}^{2+}$ (Figure 4C). 

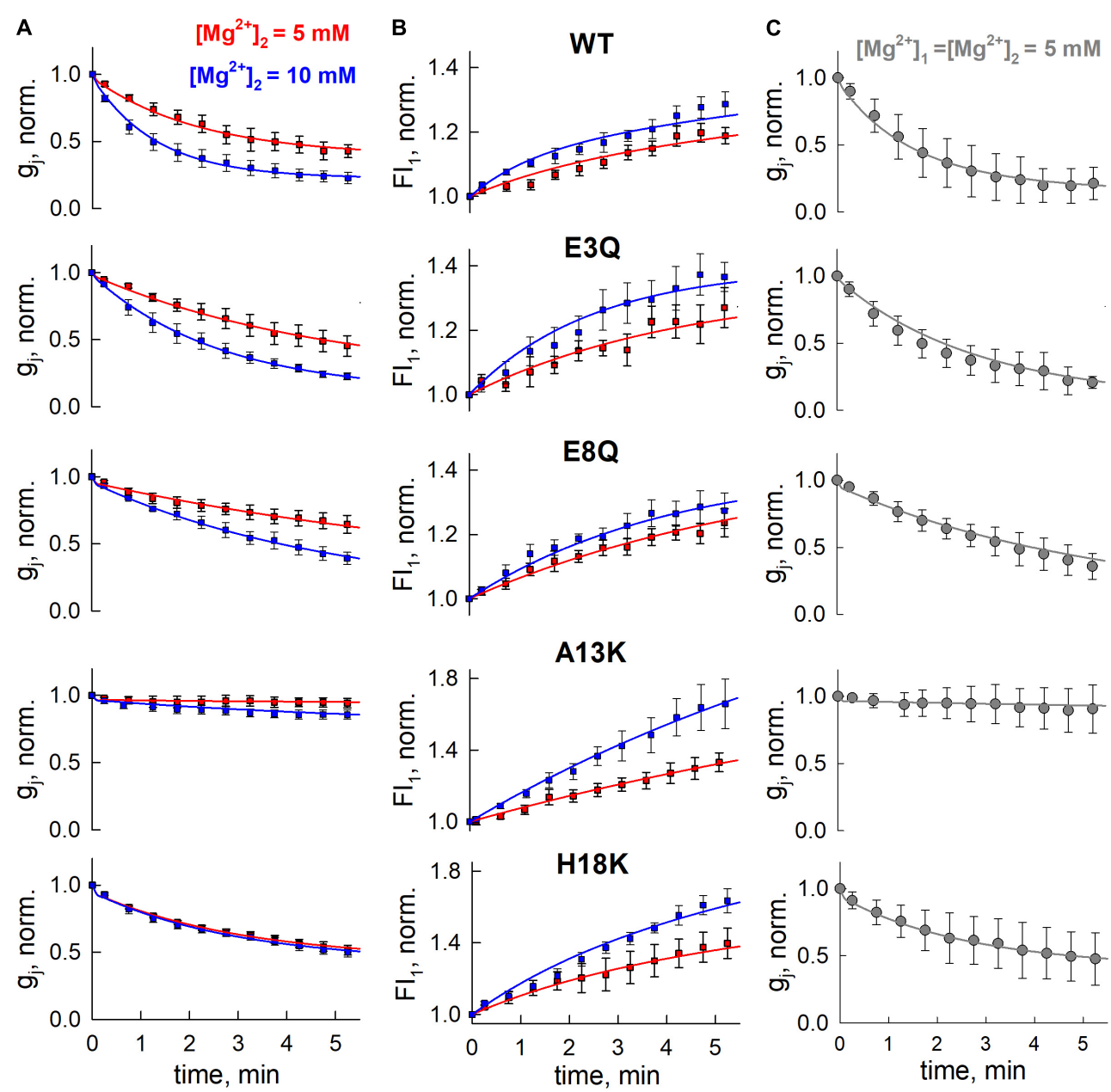

FIGURE 4 | Model fitting to data from simultaneous electrophysiological and fluorescence recordings. (A,B) Solid lines are fits to the electrophysiological (A) and fluorescence imaging (B) data presented in Figure $\mathbf{3}$ using Model 5 with Hill equation. (C) Reductions in $g_{j}$ recorded under symmetric Mg2+ conditions (both pipettes contained $5 \mathrm{mM} \mathrm{Mg}^{2+}$. Error bars denote standard deviations $(n=7)$. Data in panel $(\mathbf{C})$ was not used in the fits to obtain parameters, but only for validation of the model.

TABLE 2 | The fit and independent validation measures of different models applied to electrophysiological and fluorescent imaging data.

\begin{tabular}{|c|c|c|c|c|c|c|c|}
\hline \multirow[t]{2}{*}{ Model } & \multicolumn{2}{|c|}{ Model fitting } & \multirow{2}{*}{$\frac{\text { Validation }}{\text { SSE }}$} & \multirow[t]{2}{*}{ Model } & \multicolumn{2}{|c|}{ Model fitting } & \multirow{2}{*}{$\frac{\text { Validation }}{\text { SSE }}$} \\
\hline & $\Delta \mathrm{AIC}_{\mathrm{i}}$ & $w_{i}$ & & & $\Delta \mathrm{AIC}_{\mathrm{i}}$ & $w_{i}$ & \\
\hline Model 1 & -901.55 & 0.0000 & 0.0879 & Model 1, Hill & -902.51 & 0.0000 & 0.1017 \\
\hline Model 2 & -972.53 & 0.0000 & 0.1336 & Model 2, Hill & -941.33 & 0.0000 & 0.0552 \\
\hline Model 3 & -920.01 & 0.0000 & 0.0838 & Model 3, Hill & -914.52 & 0.0000 & 0.0869 \\
\hline Model 4 & -912.43 & 0.0000 & 0.1216 & Model 4, Hill & -1009.95 & 0.3432 & 0.0504 \\
\hline Model 5 & -993.96 & 0.0001 & 0.0669 & Model 5, Hill & -1011.25 & 0.6568 & 0.0470 \\
\hline
\end{tabular}

\section{Application of the Mathematical Model to Understand and Predict GJ Channel Behavior}

We performed computational simulations using parameters derived from the best fit model to assess how the component hemichannels in a GJ channel may be behaving in response to changes in cytoplasmic levels of $\mathrm{Mg}^{2+}$, which cannot be directly measured experimentally. As can be seen from our electrophysiological data (Figures $4 \mathbf{A}, \mathbf{C}$ ), the changes in $\mathrm{g}_{j}$ obtained using $10 \mathrm{mM} \mathrm{Mg}^{2+}$ in one patch pipette were similar to data obtained from experiments in which both patch pipettes contained $5 \mathrm{mM} \mathrm{Mg}^{2+}$. These data suggest a mechanism that allows for both hemichannels to be closed upon exposure to $\mathrm{Mg}^{2+}$ ions from their respective cytoplasmic compartments. Figure $\mathbf{5 A}$ shows simulated changes in $g_{j}$ as well as the 
TABLE 3 | The estimated parameters of Model 5 with Hill equation.

\begin{tabular}{lcccccc}
\hline Cx & $\begin{array}{c}\boldsymbol{k}_{\mathbf{1}}, \\
\left(\mathbf{m i n}^{-1}\right)\end{array}$ & $\begin{array}{c}\boldsymbol{k}_{\mathbf{2}}, \\
\left(\mathbf{m i n}^{-1}\right)\end{array}$ & $\begin{array}{c}\boldsymbol{k}_{\mathbf{3}}, \\
\left(\mathbf{m i n} \cdot \mathbf{m M}^{-1}\right)\end{array}$ & $\left.\boldsymbol{k}_{\mathbf{4}}, \mathbf{m i n}^{-1}\right)$ & $\boldsymbol{n}$ & $\begin{array}{c}\boldsymbol{P}_{\boldsymbol{\prime}} \\
\left(\mathbf{m i n} \cdot \mathbf{n S}^{-1}\right)\end{array}$ \\
\hline WT & 5.35 & 386.57 & 3.2770 & 0.1802 & 1.12 & 0.0135 \\
E3Q & 3.52 & 392.28 & 3.2366 & 0.0417 & 1.08 & 0.0258 \\
E8Q & 5.88 & 271.07 & 0.7168 & 0.0197 & 1.07 & 0.0076 \\
A13K & 3.05 & 179.34 & 0.0026 & 0.0637 & 2.84 & 0.0011 \\
H18K & 9.80 & 337.60 & 3.2391 & 0.2008 & 0.11 & 0.0072
\end{tabular}
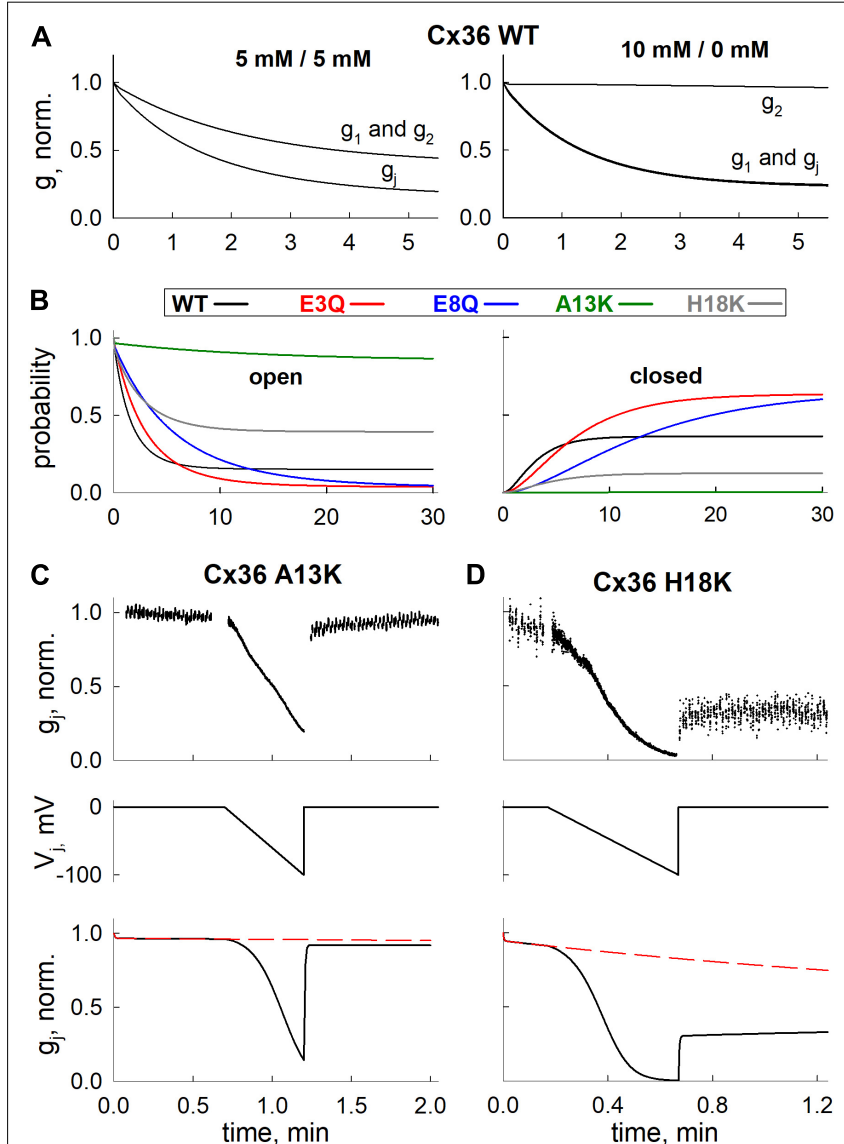

FIGURE 5 | Application of mathematical model to simulate $\mathrm{Mg}^{2+}$-induced closure of hemichannels, formed of WT and mutant Cx36. (A) Simulated $g_{j}$ decrease of Cx36 WT GJ channels ( $\left.g_{j}\right)$ and hemichannels $\left(g_{1}\right.$ and $\left.g_{2}\right)$ at different levels of $\left[\mathrm{Mg}^{2+}\right]_{\text {i. }}$ (B) Kinetics of open (i.e., both hemichannels are open) and closed (i.e., both hemichannels reside in closed, $\mathrm{Mg}^{2+}$-bound state) state probabilities of GJ channels formed of different $\mathrm{C} \times 36$ variants, simulated using $\left[\mathrm{Mg}^{2+}\right]_{1}=\left[\mathrm{Mg}^{2+}\right]_{2}=5 \mathrm{mM}$ protocol. (C,D) Experimentally measured (upper panels) and simulated (lower panels) gi decrease and recovery after application of $\mathrm{V}_{j}$ ramps (middle panels), in $\mathrm{A} 13 \mathrm{~K}$ and $\mathrm{H} 18 \mathrm{~K}$ channels. Electrophysiological recordings and simulations were performed using $\left[\mathrm{Mg}^{2+}\right]_{1}=\left[\mathrm{Mg}^{2+}\right]_{2}=5 \mathrm{mM}$ protocol.

conductances of the separate hemichannels $\left(g_{1}\right.$ and $\left.g_{2}\right)$ over a 5 min time frame. Under symmetric $5 \mathrm{mM} \mathrm{Mg}^{2+}$ conditions (left panel), both hemichannels are predicted to exhibit the same reductions in conductance, which results in a decrease in $g_{j}$ reflecting the combined closed probabilities of the hemichannels. Conversely, with $10 \mathrm{mM} \mathrm{Mg}^{2+}$ only on one side, the reduction in $g_{j}$ essentially reflects closure of the hemichannel on the high $\mathrm{Mg}^{2+}$ side, but resulted in a similar overall change in $\mathrm{g}_{j}$ as simulated with symmetric $5 \mathrm{mM} \mathrm{Mg}^{2+}$.

We also used the model to predict the changes in open and closed GJ channel probabilities under symmetric $5 \mathrm{mM} \mathrm{Mg}{ }^{2+}$ conditions over a longer time frame of $30 \mathrm{~min}$. The open and closed probabilities plotted are those in which both hemichannels are open (left panel in Figure 5B) or both hemichannels are closed in their $\mathrm{Mg}^{2+}$-stabilized closed conformations (right panel in Figure 5B); the latter information would not be directly observable from electrophysiological recordings. As in all simulations, the probabilities reflect the values at $\mathrm{V}_{\mathrm{j}}=0$. For the $\mathrm{A} 13 \mathrm{~K}$ mutation, the simulation indicates a high maintained open probability of $\sim 1.0$ even when the channel is exposed to $5 \mathrm{mM} \mathrm{Mg}^{2+}$. According to the model parameters, this high open channel probability is due to a combination of factors including a low $\mathrm{Mg}^{2+}$ permeability and, most importantly, a near absence of $\mathrm{Mg}^{2+}$ binding. H18K channels also showed a higher maintained open probability and a reduced probability that both hemichannels reside in a $\mathrm{Mg}^{2+}$-stabilized closed conformation. For these channels, however, this is mostly due to a decrease in the Hill coefficient, which resulted in a relatively lower binding rate for $\mathrm{Mg}^{2+}$ ions at high $\left[\mathrm{Mg}^{2+}\right]_{\mathrm{i}}$ concentrations. Charge substitutions at positions 3 and $8, \mathrm{E} 3 \mathrm{Q}$ and $\mathrm{E} 8 \mathrm{Q}$, resulted in reduced open probabilities and increased probabilities that both hemichannels resided in $\mathrm{Mg}^{2+}$-stabilized closed conformations, mainly due to reduced dissociation of $\mathrm{Mg}^{2+}$ from its binding site. Although the predicted open and closed probabilities of these mutants were similar at $30 \mathrm{~min}$, they differed in their kinetics, as observed experimentally on a shorter time scale.

Finally, we applied our model fit parameters to see if they can account for an experimentally observed phenomenon in Cx36 GJ channels, which is impeded recovery of $g_{j}$ in the presence of $\mathrm{Mg}^{2+}$ following channel closure promoted by applying either $\mathrm{V}_{j}$ or chemical stimuli (Palacios-Prado et al., 2013). Using a voltage ramp protocol to promote channel closure, we recorded from $\mathrm{H} 18 \mathrm{~K}$ and $\mathrm{A} 13 \mathrm{~K}$ GJs in symmetric $5 \mathrm{mM} \mathrm{Mg}^{2+}$ conditions. $\mathrm{H} 18 \mathrm{~K}$ channels retain some sensitivity to $5 \mathrm{mM} \mathrm{Mg}^{2+}$ whereas A13K channels show no sensitivity to $\mathrm{Mg}^{2+}$ altogether. Upon application of a voltage ramp, $\mathrm{H} 18 \mathrm{~K}$ channels exhibited a robust decrease in $g_{j}$. However, upon stepping back to $V_{j}=0$, there was a small, fast increase in $g_{j}$ followed by a very slow increase that resembled a plateau over a shorter time interval characteristic of the impeded recovery observed in WT Cx36 (Figure 5D). For A $13 \mathrm{~K}$ channels, which retain the ability to gate closed by $V_{j}$, there is essentially a full and rapid recovery of $g_{j}$ following application of a $\mathrm{V}_{\mathrm{j}}$ ramp (Figure 5C). Simulations using parameters for these channels obtained from fits to the data in Figure $\mathbf{4}$ are shown in Figures 5C,D. Because rate constants $k_{1}$ and $k_{2}$ can be interpreted as opening and closing rates of $\mathrm{V}_{\mathrm{j}}$-dependent gating, it follows from our previously published $\mathrm{V}_{\mathrm{j}}$-gating model (Snipas et al., 2020) that the values of $k_{1}$ and $k_{2}$ of a hemichannel should increase or decrease exponentially with $V_{j}$ (depending on the sign of $\mathrm{V}_{\mathrm{j}}$ and $\mathrm{V}_{\mathrm{j}}$-gating polarity of $\mathrm{Cx}$ isoform) during the application of $\mathrm{V}_{\mathrm{j}}$ ramp. The black solid lines (Figures 5C,D) show the predicted changes in $g_{j}$ over time when a $V_{j}$ ramp is applied with the channels exposed to symmetric $5 \mathrm{mM} \mathrm{Mg}^{2+}$ 
conditions. The dashed red lines show the predicted changes in $g_{j}$ in the absence of the $V_{j}$ ramp, reflecting changes that would occur simply upon exposure to symmetric $5 \mathrm{mM} \mathrm{Mg}^{2+}$ conditions at $\mathrm{V}_{\mathrm{j}}=0$ (i.e., transition rates $k_{1}$ and $k_{2}$ remain constant). The simulations reproduced the experimentally-observed behaviors of $\mathrm{H} 18 \mathrm{~K}$ and $\mathrm{A} 13 \mathrm{~K}$ GJ channels quite well. According to our model fitting, the impeded recovery in $\mathrm{H} 18 \mathrm{~K}$ channels is explained by increased entrance into the $\mathrm{Mg}^{2+}$-stabilized closed state promoted by the $\mathrm{V}_{\mathrm{j}}$ - induced closure. The rapid and complete recovery of $\mathrm{g}_{j}$ for $\mathrm{A} 13 \mathrm{~K}$ channels can be explained by a lack of $\mathrm{Mg}^{2+}$ binding and thus lack of a $\mathrm{Mg}^{2+}$-stabilized closed state reflected by an almost 450 -fold decrease in the ratio $k_{3} / k_{4}$ compared to WT and the other variants. In addition, the estimated ratios $k_{1} / k_{2}$, which, according to our model, should reflect the intrinsic $V_{j}$ sensitivities of the channels, corresponded well with electrophysiological data. For example, our model predicts that $\mathrm{A} 13 \mathrm{~K}$ channels should exhibit a comparable $\mathrm{V}_{\mathrm{j}}$ sensitivity to WT Cx36 channels, while H18K channels should be significantly more $V_{j}$ sensitive. The averaged $g_{j}-V_{j}$ curves obtained from voltage ramp experiments recorded at symmetric $5 \mathrm{mM} \mathrm{Mg}^{2+}$ conditions (see Supplementary Figure 1) confirm this prediction, thus providing additional support for models in which $\mathrm{Mg}^{2+}$ stabilizes hemichannel closure caused by $\mathrm{V}_{\mathrm{j}}$ gating events.

\section{Comparison of Electrostatic Potentials Between Wild Type and Mutated Cx36 Using Homology Modeling}

Given the effects we observed with charge substitutions in NT and that the NT domain constitutes the cytoplasmic vestibule of the pore, it is possible that charged residues in NT exert an electrostatic effect that reduces $\mathrm{Mg}^{2+}$ occupancy inside the pore, thereby affecting access to a binding site proposed to be within the extracellular span of the pore (Palacios-Prado et al., 2014). Thus, we evaluated the electrostatic potentials along and across the pore for WT Cx36 and each of the variants. Figures 6A,B show the corresponding maps of electrostatic potentials. Maps in Figure 6B are presented as viewed from the cytoplasmic side.

The calculated electrostatic potential profiles along the long axis of the channel (Figure 6A) show an overall negative charge bias, which was shifted more positive near the vicinities of substituted residues. Electrostatic potentials viewed in crosssections of the Cx36 channels at the locations of each of the mutated residues are shown in Figure 6B. Sums of potentials calculated inside these cross-sections of the pore are shown in Figure 6C and suggest that the substitutions resulted in substantial reductions in the negativities of the electrostatic potentials. Figure 6D shows the relationship between the reduction in negative charge inside channel pore and the change of $\mathrm{Mg}^{2+}$ permeability evaluated using our mathematical model. The data reveal a moderate negative correlation (estimated correlation coefficient was equal to -0.505) between the shifts in the electrostatic potentials and the respective ratios of the permeability coefficients. The deviations of the data points from the predicted linear relationship between these two variables (solid black line in Figure 6D) suggest that the location along the pore may be a relevant factor. That is, the $\mathrm{H} 18 \mathrm{~K}$ and A13K substitutions, which are located closest to the cytoplasm, exhibited the more robust reductions in permeability than predicted by the simple linear relationship (data points below the line). In contrast, higher than expected permeabilities (data points above the line) were observed for E8Q and E3Q variants, which are located deeper inside the channel pore.

Another feature, which is visible in Figure 6A, is an alteration in the putative shape of the pore boundary among the different variants. Some of these changes might be due to stochastic calibration of the channel structure when performing homology modeling. However, some of these changes could reflect allosteric reconfiguration of the pore-lining residues. It is likely that such alterations would have an effect on biophysical properties of the channel, such as ion permeability or ligand binding affinities.

Taken together, data using electrostatic calculations show that some of the NT substitutions can affect the surface potential, and possibly, the overall structure of the channel pore. These changes could contribute to a changed balance in ion distribution inside the channel pore, including at the putative $\mathrm{Mg}^{2+}$ binding site.

\section{DISCUSSION}

Connexin36 GJs, the principal elements of electrical synapses in the CNS, uniquely exhibit a high sensitivity to intracellular $\mathrm{Mg}^{2+}$, which represents a potentially important mechanism for mediating plasticity of electrical synaptic transmission (PalaciosPrado et al., 2013). In this study, we explored the role of the Cx36 $\mathrm{NT}$ domain in $\mathrm{Mg}^{2+}$ modulation. Although exchange between Cx36 and Cx43 sequences identified the first extracellular loop domain (E1) and the D47 residue in particular, as playing a critical role, the NT domain was subsequently implicated because of an interdependence between $\mathrm{Mg}^{2+}$ and $\mathrm{pH}$ modulation that was affected by mutations in the NT domain (Rimkute et al., 2018). Here we explicitly examined the role of $\mathrm{NT}$ on $\mathrm{Mg}^{2+}$ modulation, focusing on putative pore-lining residues spanning the length of the pore funnel, which is formed entirely by the NT domain. In keeping with the chimeric approach that exchanged $\mathrm{Cx} 36$ and $\mathrm{Cx} 43$ domains, the substitutions at the NT positions we examined were, in part, intended to convert $\mathrm{Cx} 36$ to $\mathrm{Cx} 43$ sequence. Of the 5 putative pore-lining positions examined, substitutions at 3 positions showed robust effects on $\mathrm{Mg}^{2+}$ modulation. These three substitutions, E8Q, A13K, and $\mathrm{H} 18 \mathrm{~K}$, all resulted in localized reductions in the negative charge profiles of the pore either by neutralizing a negatively charged residue or changing a neutral residue to a positively charged one. Two of the three substitutions, E8Q and H18K, resulted in GJ channels that retained sensitivity to high intracellular $\mathrm{Mg}^{2+}$, but all three substitutions eliminated the robust increase in $\mathrm{g}_{j}$ observed in $\mathrm{Cx} 36 \mathrm{GJ}$ channels exposed to low intracellular $\mathrm{Mg}^{2+}$ concentrations. These results suggest that E8Q, A13K and H18K substitutions all produce a shift toward reduced apparent affinity for intracellular $\mathrm{Mg}^{2+}$. A concomitant increase in $g_{j}$, init, the value of $g_{j}$ at the beginning of a recording, for $\mathrm{A} 13 \mathrm{~K}$ and $\mathrm{H} 18 \mathrm{~K}$ is consistent with a reduced apparent affinity and thus, reduced inhibition by $\mathrm{Mg}^{2+}$ at physiological concentrations. A13K GJ 
A

WT

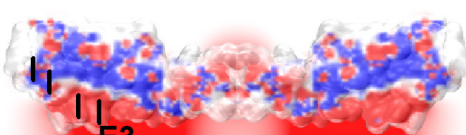
E8 E3

$\mathrm{H} 18{ }^{1}$

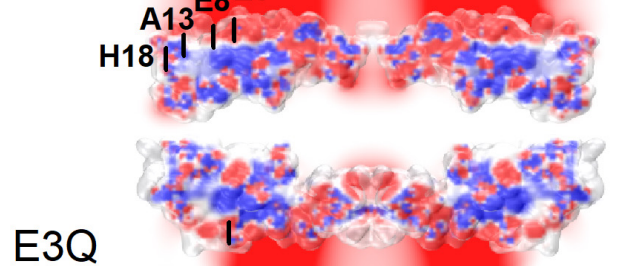

$E 3 Q$

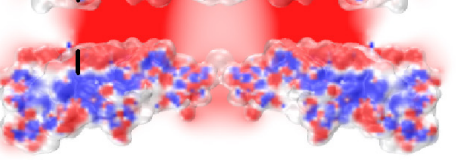

E8Q

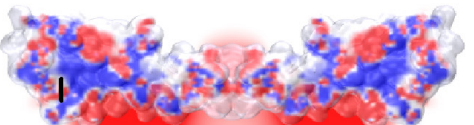

A13K
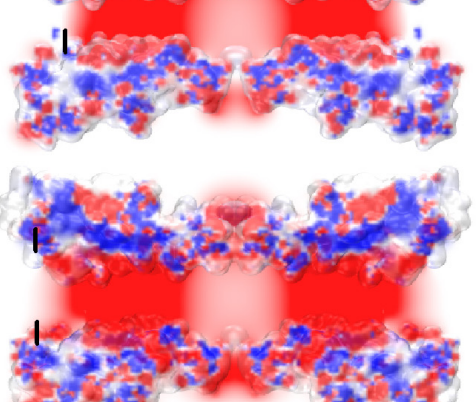

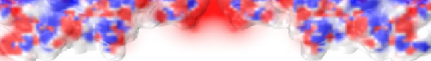

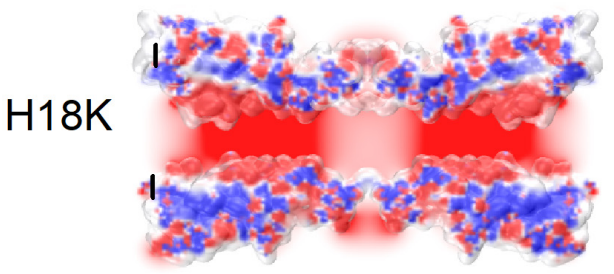

$-10$
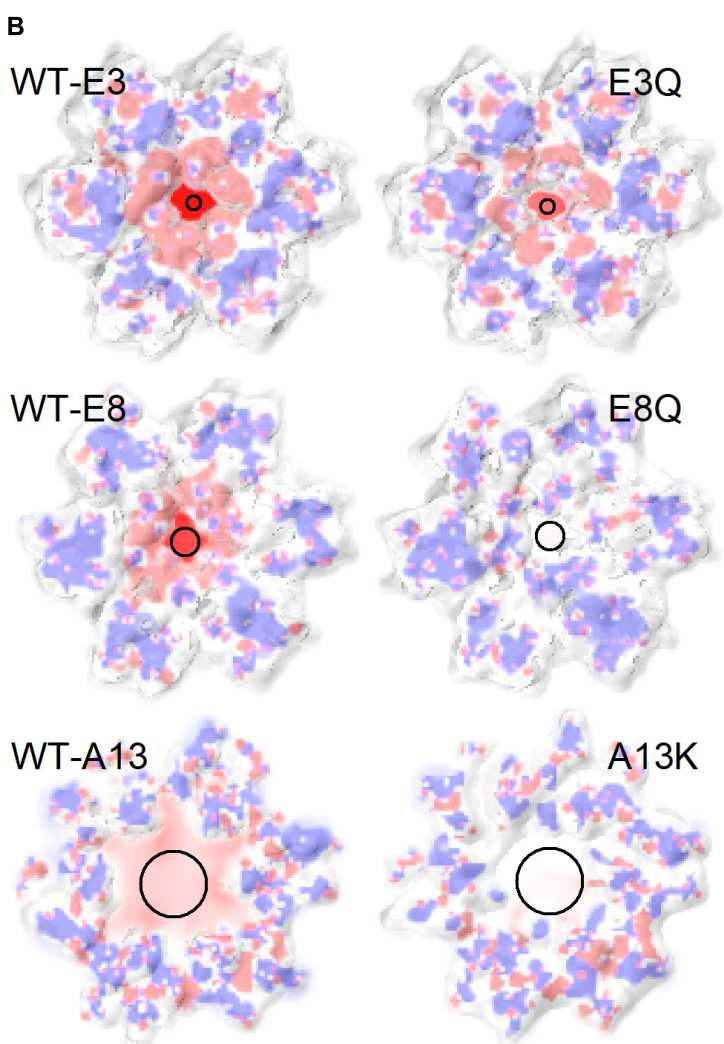

WT-H18
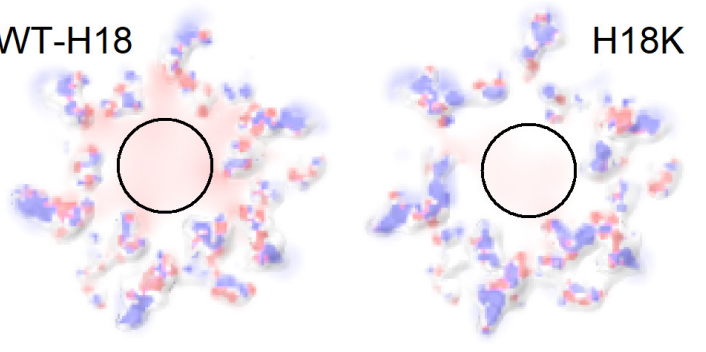

10
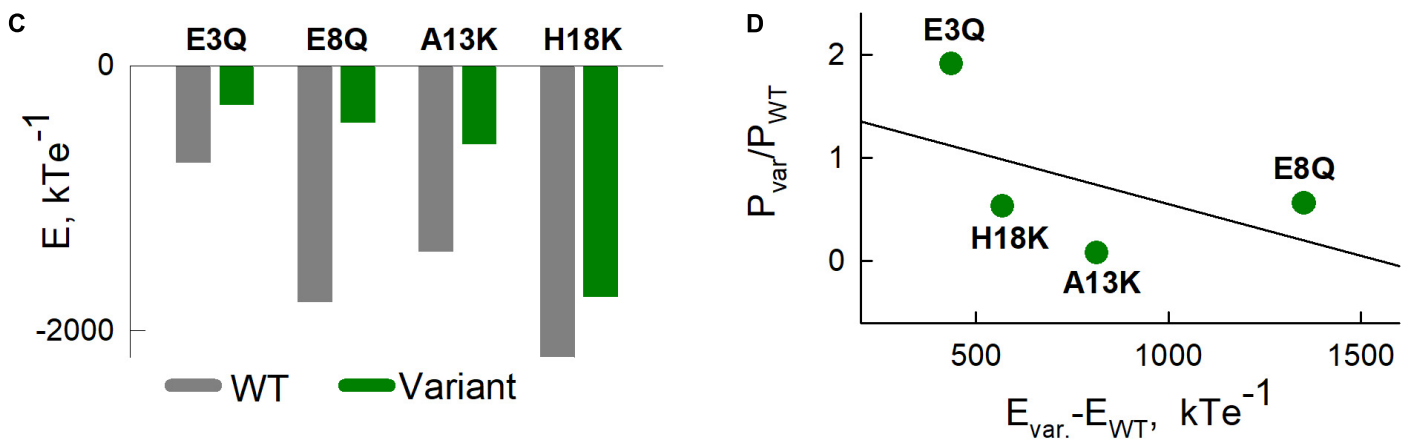

FIGURE 6 | Electrostatic potentials of Cx36 and NT variants. (A) Slices along the pore of the Cx36 showing the electrostatic potentials. Black lines indicate locations of mutated residues. (B) Cross-sections through the electrostatic potential surfaces of Cx36 WT and mutants in the vicinities of the 3rd, 8th, 13th, and 18th residues viewed from the extracellular side. In both panels (A,B), potentials were scaled between -10 and $10 \mathrm{kTe}^{-1}$ (see color scale below). (C) Sums of electrostatic potentials, calculated in the vicinities of the substituted residues. Calculations were performed by summing the average values of electrostatic potentials in $1 \times 1 \times 1$ angstrom cubes, located alongside the pore in three layers, closest to the cross-sections presented in panel (B). (D) The relationship between the differences in sums of the electrostatic potentials from panel $\mathbf{( C )}$ and the ratios of permeability coefficients $P$, estimated from Model 5 with Hill equation, for each variant. The black solid line shows a fitted linear regression curve. 
channels were distinguished by insensitivity to high intracellular $\mathrm{Mg}^{2+}$ concentrations as well, up to $10 \mathrm{mM}$, suggesting that A13K produced either a complete loss of $\mathrm{Mg}^{2+}$ binding or a very large shift in apparent affinity. Unlike charge substitutions, neutral substitutions did not result in measureable changes in modulation by $\mathrm{Mg}^{2+}$. These include neutral $\mathrm{H} 18 \mathrm{Y}$ and T5S substitutions; both are substitutions to residues present in $\mathrm{Cx} 43$. The one exception was a charge substitution at E3, E3Q, which exhibited significantly altered sensitivity only to normal $\mathrm{Mg}^{2+}$.

\section{NT and $\mathrm{Mg}^{2+}$ Sensitivity; Effects on $\mathrm{Mg}^{2+}$ Permeability and Allosteric Changes That Alter $\mathrm{Mg}^{2+}$ Binding}

To help understand the results on $\mathrm{Mg}^{2+}$ modulation, we used homology modeling, estimated electrostatic potentials within the pore center, experimentally measured $g_{j}$ and transjunctional $\mathrm{Mg}^{2+}$ fluxes and fit the electrophysiological and imaging data to mathematical models that took into account GJ channel gating and $\mathrm{Mg}^{2+}$ binding to open and/or closed states. $\mathrm{Mg}^{2+}$ flux data along with simultaneous measurements of $g_{j}$, enabled assessment of total junctional membrane permeability normalized to the junctional conductance, thereby providing an estimate of open channel permeability to $\mathrm{Mg}^{2+}$.

Model fitting of the data showed that Model 5 with the Hill equation for $\mathrm{Mg}^{2+}$ binding provided the best fit according to values of AIC. The estimated Akaike weights (see Table 2) indicate that Model 5 with the Hill equation is almost twice as likely to be the best compared to the second best model, Model 4 with the Hill equation (see Table 2). In the latter model, hemichannel closure is caused by $\mathrm{Mg}^{2+}$ binding to the open state (the first step), which is followed by a $\mathrm{Mg}^{2+}$ independent transition to a second closed state (the second step). However, the estimated values of the parameters for Model 4 predict large differences between WT and some of the variants, such as E3Q (see Supplementary Material), which is not supported by the moderate differences observed experimentally (Figures 2-4). In addition, the Model 5 provided the lowest SSE for independent validation data on reductions in $g_{j}$ in high symmetric $\mathrm{Mg}^{2+}$ concentrations, which was not included into the model fitting and analyses of AIC values. Thus, the combination of model fitting and independent data validation strongly indicates that Model 5 with Hill equation is the best candidate among the considered models.

Model fits indicate that the $\mathrm{A} 13 \mathrm{~K}$ variant is the only one that shows a substantive, $\sim 10$-fold, reduction in $\mathrm{Mg}^{2+}$ permeability, which could contribute significantly to a reduced apparent affinity for $\mathrm{Mg}^{2+}$ binding. All the charge substitutions resulted in reduced negativity of the electrostatic profiles, and these changes correlated with the changes in $\mathrm{Mg}^{2+}$ permeability. However the correlation was only moderate, which indicates that changes in $\mathrm{Mg}^{2+}$ permeability are affected by other factors. For example, Figure $6 \mathrm{D}$ shows that $\mathrm{A} 13 \mathrm{~K}$ and $\mathrm{H} 18 \mathrm{~K}$, which are located closer to the cytoplasm than $\mathrm{E} 3 \mathrm{Q}$ and $\mathrm{E} 8 \mathrm{Q}$, exhibited a more robust decrease in $\mathrm{Mg}^{2+}$ permeability than would be predicted by a simple linear dependence on the reduced negativity.

Given that reduced $\mathrm{Mg}^{2+}$ permeability alone cannot explain the robust effects of NT substitutions on $\mathrm{Mg}^{2+}$ sensitivity, likely explanations include altered $\mathrm{Mg}^{2+}$ binding and/or altered stabilization of the $\mathrm{Mg}^{2+}$-bound closed state. As previously indicated, studies reported D47 in the E1 domain as an important component of a putative $\mathrm{Mg}^{2+}$ binding site (Palacios-Prado et al., 2014). In a GJ channel, this position in each hemichannel would be located deep in the pore, in the extracellular gap region, remote from NT. In order for NT substitutions to impact $\mathrm{Mg}^{2+}$ binding at D47, structural changes would need to translate from NT to E1. Early NMR studies of NT peptides composed of Cx32 sequence indicated that the NT domain has a helical component in its N-terminal end and forms on open turn in the vicinity of the G12 residue (Purnick et al., 2000a). This NT structure exhibited intrinsic flexibility that could serve to position the initial helical portion of the NT domain into the pore. Although this characterization was based on NMR studies of isolated NT peptides, the fundamental arrangement of the NT domain containing a turn was later confirmed by high resolution structures of Cx26 and Cx46/Cx50 GJ channels (Maeda et al., 2009; Myers et al., 2018) as depicted in the homology model for $\mathrm{Cx} 36$ in Figure 1. The turn that positions the N-terminal end of NT into the pore also explains the reported contributions of NT to voltage sensing (Verselis et al., 1994; Purnick et al., 2000b), which in GJs is mediated by residues positioned in the pore where they can sense the transjunctional field independent of the transmembrane field (Harris et al., 1981; Bukauskas and Verselis, 2004; Bargiello et al., 2018). Channel closure was associated with movement of the pore-forming region of NT toward the cytoplasm. The ultimate picture that has emerged is an NT structure that is dynamic and that participates in conformational changes that open and close GJ channels in response to transjunctional voltage and perhaps other stimuli. Although not yet understood in structural detail, a completed and equilibrated structure of a Cx26 hemichannel refined by molecular dynamics simulations suggested that gating of $\mathrm{Cx}$ channels involves rearrangement of a network of residues that interact electrostatically and through van der Waals forces to impact the positioning of a parahelical domain residing in a segment of E1. Based on a more recent cryo-EM structure of a Cx46/50 GJ channel resolved at a resolution of $1.9 \AA$ (Flores et al., 2020), all-atom molecular dynamics simulations applied to a T39R cataract mutant in Cx50, which leads to increased hemichannel opening, suggested that stabilization of the open state of the hemichannel could occur through a direct interaction between NT and TM1, specifically through two alternative configurations, a D3-T39R salt bridge and a tripartite interaction involving G2, T39R and E42 (Tong et al., 2021). Thus, what is apparent is that NT and E1 domains are highly coupled, potentially through a combination of allosteric and direct interactions, that can plausibly link changes in NT with $\mathrm{Mg}^{2+}$ binding putatively assigned to reside in $\mathrm{E} 1$.

We note that a G46D charge substitution in Cx43, which aligns with D47 in Cx36, was able to confer a high $\mathrm{Mg}^{2+}$ sensitivity to Cx43 despite an NT domain that naturally contains a positively charged Lys at position 13. Thus, the effects of NT residues would appear to differ between $\mathrm{Cx} 43$ and $\mathrm{Cx} 36$, which is not surprising given that sequence differences between these Cxs likely result in differences in the patterns of residues comprising 
the electrostatic and van der Waals networks that govern $\mathrm{Cx}$ channel gating and likely contribute to the coupling of the NT and E1 domains. Conversely, the presence of charge alone at the D47 position in other Cxs does not necessarily confer $\mathrm{Mg}^{2+}$ sensitivity, again consistent with the integrative nature of the conformational changes that occur with Cx channel opening and closing. Moreover, the pore boundaries and overall electrostatic profiles of $\mathrm{Cx}$ channels are likely to differ, evident from the available structures for Cx26 and Cx46/Cx50 (Maeda et al., 2009; Myers et al., 2018), which can contribute to differences in $\mathrm{Mg}^{2+}$ permeability. In this view, $\mathrm{Cx}$ channels, although related in overall construction, likely differ in the positioning of residues within the network and the pore, explaining the wide heterogeneity in biophysical properties documented among various $\mathrm{Cx}$ channels and possible differential effects of individual amino acid substitutions in different $\mathrm{Cx}$ channels.

Although homology modeling using MODELLER and the available crystal structures of $\mathrm{Cx} 26$ and $\mathrm{Cx} 46 / 50$ provides a viable and established method for approximating the structure of $\mathrm{Cx} 36$, other algorithms are available to test alternative structural predictions for $\mathrm{Cx} 36$. For example, the protein folding algorithm AlphaFold (Senior et al., 2020) predicts a Cx36 NT domain that adopts a wide bend that does not pack well with TM1/E1 residues. This orientation is unlike the NT domains in the Cx26 and $\mathrm{Cx} 46 / 50$ crystal structures as well as in the NT domains of other $\mathrm{Cx}$ structures predicted by AlphaFold, in which the bend angle of the NT domain is steep allowing for interactions with TM1/E1 residues. Whether this difference is indicative of a unique conformational structure of $\mathrm{Cx} 36$ remains unknown. We note that AlphaFold shows that the predicted structure near the 13-14 positions in NT, which is the putative hinge region of the bend, shows low reliability (only $\sim 50$ percent confidence level). Also, although chimeric studies in which the NT domain of $\mathrm{Cx} 36$ was substituted with $\mathrm{Cx} 43$ sequence produced functional channels, the reciprocal chimera, in which NT domain of Cx43 was substituted with $\mathrm{Cx} 36$ sequence failed to yield functional channels suggesting that there may be a sufficiently different NT structure for $\mathrm{Cx} 36$ such that it fails to be accommodated properly into a $\mathrm{Cx} 43$ background. In the event that the Cx36 NT structure adopts a unique orientation compared to other Cxs, the effects of the NT substitutions we examined here will be informative in providing mechanistic insights into NT structure and function.

Our modeling did not explicitly reveal changes in potential interactions between the residues at the substituted sites or at D47. However, our homology modeling was based on the Cx46/Cx50 structure, which is proposed to be in the open state, with the NT domain stabilized by anchoring hydrophobic interactions with TM1/TM2 helices (Myers et al., 2018; Yue et al., 2021). Thus, if $\mathrm{Mg}^{2+}$ binding occurs in the closed conformation, as suggested by our mathematical modeling data, we should not expect to detect any potential interactions in the homology model. Moreover, given the critical dependence on the structural template, it is likely that structural perturbations and alterations in side chain packing resulting from sequence divergence between $\mathrm{Cx} 36$ and $\mathrm{Cx} 46 / \mathrm{Cx} 50$ would be lost in the approximated channel structure. Although Cxs can exhibit considerable sequence similarities, particularly in the more conserved NT, transmembrane and extracellular loop domains, phylogenetic analyses have divided Cxs into three main groups, with Cx36 belonging to the delta or Group IIIa designation (White et al., 2004; Cruciani and Mikalsen, 2007). Available structures Cx46/Cx50 and Cx26 represent Group I (Cx46/Cx50) and Group II (Cx26) connexins and, thus are more distant to $\mathrm{Cx} 36$. We chose to use the $\mathrm{Cx} 46 / \mathrm{Cx} 50$ structure as a template due to a better estimated sequence similarity of more than 53 percent.

\section{Insights About $\mathrm{Mg}^{2+}-$ Mediated Regulation of Cx36 Channels From Modeling Data}

Electrophysiological studies of Cx36 GJ channels are hindered by their unusually low unitary conductance, which precludes direct measurement of open channel conductance and open probability. Our data showed that the same applies for all the Cx36 variants considered in this study. Thus, we combined macroscopic electrophysiological recordings with mathematical modeling to gain insights into channel behavior. Using different kinetic schemes of $\mathrm{Mg}^{2+}$-mediated channel gating, E8Q, A13K and $\mathrm{H} 18 \mathrm{~K}$ GJ channels were consistently predicted to be less permeable to $\mathrm{Mg}^{2+}$ compared to WT Cx36. The models also suggest that the observed differences in $\mathrm{Mg}^{2+}$-mediated changes in $g_{j}$ can be adequately explained by a 3 -state linear scheme. The best fit to the data was provided by the model in which the first transition represents $\mathrm{Mg}^{2+}$-independent gating between open and closed states in each of the hemichannels, and the second transition represents the binding of $\mathrm{Mg}^{2+}$ and stabilization of the closed hemichannel conformation. The estimated values of the transition rates associated with the $\mathrm{Mg}^{2+}$-independent transition (Table 2) suggest that it represents stochastic gating and can occur even in absence of $\mathrm{V}_{\mathrm{j}}$ gradients. Such stochastic gating was demonstrated for $\mathrm{Cx} 26$ hemichannels for which single channel data is available (Sanchez et al., 2013). Modeling of data using our recently published gating model, $4 \mathrm{SM}$, that takes into account contingent gating of two series hemichannels and the distribution of $\mathrm{Vj}$ across each hemichannel (Snipas et al., 2020) indicates that such stochastic gating at $V_{j}=0 \mathrm{mV}$ would be sufficient to drive a $\mathrm{Mg}^{2+}$-dependent reduction in $g_{j}$ caused by $\mathrm{Mg}^{2+}$ binding to the closed state. Moreover, as mentioned in the results, the estimated values of model parameters $k_{1}$ and $k_{2}$ of different variants correlated well with the observed changes in $\mathrm{V}_{\mathrm{j}}$ sensitivity at high $\mathrm{Mg}^{2+}$ concentrations. This provides additional support for our model, in which $\mathrm{Mg}^{2+}$ ions stabilize hemichannel closure induced by the intrinsic $\mathrm{V}_{\mathrm{j}}$-dependent gating. Interestingly, this scenario is very similar to the collapsed version of the mathematical model for the effects of $\mathrm{Ca}^{2+}$ on Cx46 hemichannels, in which $\mathrm{Ca}^{2+}$ was proposed to bind to and stabilize the closed conformation and in which $\mathrm{Ca}^{2+}$ binding is allosterically coupled to the voltage sensor (Pinto et al., 2017). Of note, the effects of $\mathrm{Mg}^{2+}$ on Cx46 hemichannels are similar to $\mathrm{Ca}^{2+}$, but with lower affinity.

Thus, Model 5 provides the best overall fit to the data on $\mathrm{V}_{\mathrm{j}}$ gating and the observed $\mathrm{Mg}^{2+}$-induced changes in $\mathrm{g}_{\mathrm{j}}$ among the considered candidate models. A simpler, two-state $O \leftrightarrow C$ kinetic scheme together with the Hill equation to describe $\mathrm{Mg}^{2+}$ 
binding provided a good fit as well, but it could not reproduce the impeded recovery of $g_{j}$ (data not shown). We acknowledge that whether $\mathrm{Mg}^{2+}$ binds to the closed and not to the open conformation of Cx36 GJ channels lacks direct experimental evidence. However, our model fitting together with data on $\mathrm{Cx} 46$ regulation by $\mathrm{Ca}^{2+}$ strengthens this possibility and may represent a common mechanism by which Cxs are regulated by divalent cations.

Given that we could not measure single channel events for Cx36 GJs, we cannot exclude effects of $\mathrm{Mg}^{2+}$ on unitary conductance. Intracellular $\mathrm{Mg}^{2+}$ was shown to affect unitary conductance of Cx50 GJ channels (Tejada et al., 2018). However, the changes over a 100 -fold range of $\mathrm{Mg}^{2+}$ concentrations were relatively small and would not account for the effects observed for Cx36 GJs. In any case, we were unable to measure unitary events for $\mathrm{A} 13 \mathrm{~K}$ channels, which lack $\mathrm{Mg}^{2+}$ modulation, indicating the inherently small conductance characteristic of WT Cx36 was retained in A13K channels. Moreover, this issue was addressed in a study by Palacios et al. (Palacios-Prado et al., 2014), by taking advantage of a $\mathrm{Cx} 43 / \mathrm{Cx} 36$ chimera with a large unitary conductance in which $\mathrm{Mg}^{2+}$ sensitivity was transferred by domain substitution. In that study, $\mathrm{Mg}^{2+}$ was shown to have no effect on unitary conductance, despite strong modulation of $\mathrm{g}_{\text {j }}$ indicating that $\mathrm{Mg}^{2+}$ acts by modulating channel gating. For these reasons, in this study, we only considered kinetic schemes in which hemichannel transits between fully open and closed states.

$\mathrm{Mg}^{2+}$-stabilization of the closed conformation requires access to the binding site when the channel is closed. All-atom molecular dynamics (MD) simulations of Cx26 hemichannels based on the Cx26 structure suggested that the reorganization of the electrostatic and van der Waals networks that is proposed to represent gating results in a substantial narrowing of the pore in the vicinity of the parahelical region in E1, which in Cx26 spans from E42 to F51 (Kwon et al., 2011; Bargiello et al., 2018). Assuming a conserved gating motif in all Cxs including Cx36, a constriction could occur near the putative $\mathrm{Mg}^{2+}$ binding site potentially allowing for such a state-dependent binding event. These MD simulations also suggested that the reorganization of the network could change the angle of the bend in the TM1/E1 region thereby changing the conformation at the cytoplasmic entrance, which could represent an aspect of the coupling between $\mathrm{NT}$ and $\mathrm{E} 1$ regions and thus the ability of NT and E1 mutations to affect $\mathrm{Mg}^{2+}$ sensitivity.

\section{The Existence of Hypothetical Deep-Closed State}

Previous studies of $\mathrm{Mg}^{2+}$ modulation of Cx36 suggested that there may be a "deep-closed" conformation, in which both hemichannels of a Cx36 GJ channel are in a closed conformation resulting in the trapping of $\mathrm{Mg}^{2+}$ ions inside a pocket between two physical gates (Palacios-Prado et al., 2013). Such a scenario could leave Cx36 GJ channels in a stable and long-lived closed conformation. Our simulation data in Figure 5B showed that the probability of residing in a double-closed state (both hemichannels closed) is relatively high for Cx36 and most of the variants we studied, even in the absence of a trapping mechanism. However, when we simulated such a mechanism into our model by significantly reducing $k_{4}$ transition rates when both hemichannels reached a $\mathrm{Mg}^{2+}$-bound closed state, the probability to reach a double-closed state exceeded $90 \%$ during 2-3 hours of simulation, even at physiological concentrations of $\mathrm{Mg}^{2+}$. Given that the half-life of a Cx36 GJ channel can be short and on the order of 3 hrs (Wang et al., 2015), such a hypothetical mechanism could provide an explanation, in part, for the generally low fraction of channels within a GJ plaque that appear to be functional at any given time, particularly those formed of Cx36 (Curti et al., 2012; Marandykina et al., 2013; Szoboszlay et al., 2016). Given that we did not observe a larger plaque size associated with A13K GJs (data not shown), which lack $\mathrm{Mg}^{2+}$-induced stabilization of the closed conformation, they may exhibit a higher fraction of functional channels, a possibility consistent with a significantly higher value for $\mathrm{g}_{\mathrm{j}}$,init.

\section{NT Mutations}

To our knowledge, there are no $\mathrm{Cx} 36$ mutations associated with disease that have been identified. However, mutations in 10 different human Cxs have been linked to over two dozen distinct genetic disorders with eight of them linked to mutations in $\mathrm{Cx} 26$ alone (Delmar et al., 2018; Srinivas et al., 2018). Of particular interest here, several Cx26 disease-causing missense mutations that result in syndromic forms of deafness have been shown to cluster in the NT and E1 domains. These mutant channels have been shown to function, either as hemichannels and/or as GJ channels, and exhibit a spectrum of aberrant functional properties (Lee and White, 2009; Sanchez and Verselis, 2014). Mutation at the G12 position in NT of Cx32 has been reported in association with Charcot-Marie-Tooth disease (Bergoffen et al., 1993) and in Cx30.3 with erythrokeratodermia variabilis (Richard et al., 1998). Mutations at several NT positions in Cx46 are associated with congenetical cataractogenesis (Berthoud and Ngezahayo, 2017). Given the structural configuration of the NT domain as the cytoplasmic vestibule of the pore, it is not surprising that NT mutations, through altered intra- and intersubunit interactions, can alter the structure of NT and its inherent flexibility, thereby imparting longer-range effects in other parts of the channel. For $\mathrm{Cx} 36$, the interdependence between $\mathrm{pH}$ and $\mathrm{Mg}^{2+}$ modulation could be mediated by titration of NT residues that plausibly cause allosteric changes that affect the binding of $\mathrm{Mg}^{2+}$, putatively ascribed to the E1 domain, and/or by alterations in gating that affect the state-dependent nature of the binding of $\mathrm{Mg}^{2+}$ suggested by our modeling. Absent mutations, posttranslational modifications, such as acetylation of the N-terminal methionine that was suggested as mechanism for imparting differential permeability characteristics to $\mathrm{Cx} 26$ (Kwon et al., 2011), may also play a role in Cx36. Phosphorylation of $\mathrm{Cx} 36$ residues in cytoplasmic domains have been shown to occur in association with CaMKII and $\mathrm{Ca}^{2+}$ influx and to lead to increases the strength of electrical coupling (Del Corsso et al., 2012; Siu et al., 2021). Subsequent effects on $\mathrm{Mg}^{2+}$ sensitivity by phosphorylation by CaMKII or other posttranslational modifications can also occur, thereby potentially linking $\mathrm{Mg}^{2+}$ modulation to neuronal activity and the metabolic status of the brain. 


\section{DATA AVAILABILITY STATEMENT}

The raw data supporting the conclusions of this article will be made available by the authors, without undue reservation.

\section{AUTHOR CONTRIBUTIONS}

TK, LK, LG, MS, NP-P, and VV designed the research and analyzed the data. TK, LG, and LK performed the experiments. TK performed the homology modeling and molecular dynamic simulations. MS constructed and applied mathematical models. LG, LK, and NP-P critically revised the manuscript. TK, MS, and VV wrote the manuscript. All authors contributed to the article and approved the submitted version.

\section{REFERENCES}

Ainscow, E. K., Mirshamsi, S., Tang, T., Ashford, M. L., and Rutter, G. A. (2002). Dynamic imaging of free cytosolic ATP concentration during fuel sensing by rat hypothalamic neurones: evidence for ATP-independent control of ATPsensitive $\mathrm{K}(+)$ channels. J. Physiol. 544, 429-445. doi: 10.1113/jphysiol.2002. 022434

Andrasi, E., Igaz, S., Molnar, Z., and Mako, S. (2000). Disturbances of magnesium concentrations in various brain areas in Alzheimer's disease. Magnes. Res. 13, 189-196.

Barbagallo, M., and Dominguez, L. J. (2007). Magnesium metabolism in type 2 diabetes mellitus, metabolic syndrome and insulin resistance. Arch. Biochem. Biophys. 458, 40-47. doi: 10.1016/j.abb.2006.05.007

Barbiroli, B., Iotti, S., Cortelli, P., Martinelli, P., Lodi, R., Carelli, V., et al. (1999). Low brain intracellular free magnesium in mitochondrial cytopathies. J. Cereb. Blood Flow Metab. 19, 528-532. doi: 10.1097/00004647-199905000-00007

Bargiello, T. A., Oh, S., Tang, Q., Bargiello, N. K., Dowd, T. L., and Kwon, T. (2018) Gating of Connexin Channels by transjunctional-voltage: conformations and models of open and closed states. Biochim. Biophys. Acta Biomembr. 1860, 22-39. doi: 10.1016/j.bbamem.2017.04.028

Bedner, P., Niessen, H., Odermatt, B., Kretz, M., Willecke, K., and Harz, H. (2006). Selective Permeability of Different Connexin Channels to the Second Messenger Cyclic AMP. J. Biol. Chem. 28, 6673-6681. doi: 10.1074/jbc.M511235200

Bennett, M. V., and Zukin, R. S. (2004). Electrical coupling and neuronal synchronization in the Mammalian brain. Neuron 41, 495-511. doi: 10.1016/ s0896-6273(04)00043-1

Bergoffen, J., Scherer, S., Wang, S., Scott, M. O., Bone, L., Paul, D., et al. (1993). Connexin mutations in X-linked Charcot-Marie-Tooth disease. Science 262, 2039-2042. doi: 10.1126/science.8266101

Berthoud, V. M., and Ngezahayo, A. (2017). Focus on lens connexins. BMC Cell Biol. 18:6. doi: 10.1186/s12860-016-0116-6

Bissiere, S., Zelikowsky, M., Ponnusamy, R., Jacobs, N. S., Blair, H. T., and Fanselow, M. S. (2011). Electrical synapses control hippocampal contributions to fear learning and memory. Science 331, 87-91. doi: 10.1126/science.1193785

Bukauskas, F. F., and Verselis, V. K. (2004). Gap junction channel gating. Biochim. Biophys. Acta 1662, 42-60. doi: 10.1016/j.bbamem.2004.01.008

Cascio, M. (2005). Connexins and their environment: effects of lipids composition on ion channels. Biochim. Biophys. Acta 1711, 142-153. doi: 10.1016/j.bbamem. 2004.12.001

Connors, B. W., and Long, M. A. (2004). Electrical synapses in the mammalian brain. Annu. Rev. Neurosci. 27, 393-418. doi: 10.1146/annurev.neuro.26. 041002.131128

Cruciani, V., and Mikalsen, S. O. (2007). Evolutionary selection pressure and family relationships among connexin genes. Biol. Chem. 388, 253-264. doi: 10.1515/ BC. 2007.028

\section{FUNDING}

This research was funded by European Social Fund (project No 09.3.3-LMT-K-712-02-0073) under grant agreement with the Research Council of Lithuania (LMTLT), as well as by the Research and Innovation Fund of Kaunas University of Technology (project grant No. PP22/182) and the Research Fund of Lithuanian University of Health Sciences, and partially funded by the Fondo Nacional de Desarrollo Científico y Tecnológico (FONDECYT) grant number 3180272 to NP-P.

\section{SUPPLEMENTARY MATERIAL}

The Supplementary Material for this article can be found online at: https://www.frontiersin.org/articles/10.3389/fphys. 2022.839223/full\#supplementary-material

Curti, S., Hoge, G., Nagy, J. I, and Pereda, A. E. (2012). Synergy between electrical coupling and membrane properties promotes strong synchronization of neurons of the mesencephalic trigeminal nucleus. J. Neurosci. 32, 4341-4359. doi: 10.1523/JNEUROSCI.6216-11.2012

Del Corsso, C., Iglesias, R., Zoidl, G., Dermietzel, R., and Spray, D. C. (2012). Calmodulin dependent protein kinase increases conductance at gap junctions formed by the neuronal gap junction protein connexin36. Brain Res. 1487, 69-77. doi: 10.1016/j.brainres.2012.06.058

Delmar, M., Laird, D. W., Naus, C. C., Nielsen, M. S., Verselis, V. K., and White, T. W. (2018). Connexins and Disease. Cold Spring Harb Perspect Biol. 10:a029348. doi: 10.1101/cshperspect.a029348

Dolinsky, T. J., Nielsen, J. E., Mccammon, J. A., and Baker, N. A. (2004). PDB2PQR: an automated pipeline for the setup of Poisson-Boltzmann electrostatics calculations. Nucleic Acids Res. 32, W665-W667. doi: 10.1093/nar/gkh381

Dworak, M., Mccarley, R. W., Kim, T., Kalinchuk, A. V., and Basheer, R. (2010). Sleep and brain energy levels: ATP changes during sleep. J. Neurosci. 30, 9007-9016. doi: 10.1523/JNEUROSCI.1423-10.2010

Fang, B., Wang, D., Huang, M., Yu, G., and Li, H. (2010). Hypothesis on the relationship between the change in intracellular $\mathrm{pH}$ and incidence of sporadic Alzheimer's disease or vascular dementia. Int. J. Neurosci. 120, 591-595. doi: $10.3109 / 00207454.2010 .505353$

Flores, J. A., Haddad, B. G., Dolan, K. A., Myers, J. B., Yoshioka, C. C., Copperman, J., et al. (2020). Connexin-46/50 in a dynamic lipid environment resolved by CryoEM at 1.9 A. Nat. Commun. 11:4331. doi: 10.1038/s41467-020-18120-5

González-Nieto, D., Gómez-Hernández, J. M., Larrosa, B., Gutiérrez, C., Muñoz, M. D., Fasciani, I., et al. (2008). Regulation of neuronal connexin-36 channels by pH. Proc. Natl. Acad Sci. U.S.A. 105, 17169-17174. doi: 10.1073/pnas. 0804189105

Harris, A. L., Spray, D. C., and Bennett, M. V. L. (1981). Kinetic properties of a voltage-dependent junctional conductance. J. Gen. Physiol. 77, 95-117. doi: 10.1085/jgp.77.1.95

Hinsberger, A. D., Williamson, P. C., Carr, T. J., Stanley, J. A., Drost, D. J., Densmore, M., et al. (1997). Magnetic resonance imaging volumetric and phosphorus 31 magnetic resonance spectroscopy measurements in schizophrenia. J. Psychiat. Neurosci. 22, 111-117.

Int Veld, P. A., Pipeleers, D. G., and Gepts, W. (1986). Glucose alters configuration of gap junctions between pancreatic islet cells. Am. J. Physiol. 251, C191-C196. doi: 10.1152/ajpcell.1986.251.2.C191

Kwon, T., Harris, A. L., Rossi, A., and Bargiello, T. A. (2011). Molecular dynamics simulations of the Cx26 hemichannel: evaluation of structural models with Brownian dynamics. J. Gen. Physiol. 138, 475-493. doi: 10.1085/jgp.201110679

Lee, J., Patel, D. S., Ståhle, J., Park, S.-J., Kern, N. R., Kim, S., et al. (2018). CHARMM-GUI membrane builder for complex biological membrane simulations with glycolipids and lipoglycans. J. Chem. Theory Comput. 15, 775-786. doi: 10.1021/acs.jctc.8b01066 
Lee, J. R., and White, T. W. (2009). Connexin-26 mutations in deafness and skin disease. Expert Rev. Mol. Med. 11:e35. doi: 10.1017/S1462399409001276

Locke, D., and Harris, A. L. (2009). Connexin channels and phospholipids: association and modulation. BMC Biol. 7:52. doi: 10.1186/1741-7007-7-52

Luthi, D., Gunzel, D., and Mcguigan, J. A. (1999). Mg-ATP binding: its modification by spermine, the relevance to cytosolic $\mathrm{Mg} 2+$ buffering, changes in the intracellular ionized $\mathrm{Mg} 2+$ concentration and the estimation of $\mathrm{Mg} 2+$ by 31P-NMR. Exp. Physiol. 84, 231-252. doi: 10.1111/j.1469-445x.1999.01 799.x

Maeda, S., Nakagawa, S., Suga, M., Yamashita, E., Oshima, A., Fujiyoshi, Y., et al. (2009). Structure of the connexin 26 gap junction channel at 3.5 A resolution. Nature 458, 597-602. doi: 10.1038/nature07869

Marandykina, A., Palacios-Prado, N., Rimkutë, L., Skeberdis, V. A., and Bukauskas, F. F. (2013). Regulation of Connexin-36 Gap Junction Channels by n-Alkanols and Arachidonic Acid. J. Physiol. 591, 2087-2101. doi: 10.1113/jphysiol.2013. 250910

Matsuda, H., Kurata, Y., Oka, C., Matsuoka, S., and Noma, A. (2010). Magnesium gating of cardiac gap junction channels. Prog. Biophys. Mol. Biol. 103, 102-110. doi: 10.1016/j.pbiomolbio.2010.05.009

Meda, P., Perrelet, A., and Orci, L. (1979). Increase of gap junctions between pancreatic B-cells during stimulation of insulin secretion. J. Cell Biol. 82, 441-448. doi: 10.1083/jcb.82.2.441

Myers, J. B., Haddad, B. G., O'neill, S. E., Chorev, D. S., Yoshioka, C. C., Robinson, C. V., et al. (2018). Structure of native lens connexin $46 / 50$ intercellular channels by cryo-EM. Nature 564, 372-377. doi: 10.1038/s41586-018-0786-7

Neijssen, J., Herberts, C., Drijfhout, J. W., Reits, E., Janssen, L., and Neefjes, J. (2005). Cross-presentation by intercellular peptide transfer through gap junctions. Nature 434, 83-88. doi: 10.1038/nature03290

Niessen, H., Harz, H., Bedner, P., Kramer, K., and Willecke, K. (2000). Selective permeability of different connexin channels to the second messenger inositol 1,4,5-trisphosphate. J. Cell Sci. 113, 1365-1372. doi: 10.1242/jcs.113.8.1365

Noma, A., and Tsuboi, N. (1987). Dependence of junctional conductance on proton, calcium and magnesium ions in cardiac paired cells of guinea-pig. J. Physiol. 382, 193-211. doi: 10.1113/jphysiol.1987.sp016363

Orci, L., Unger, R. H., and Renold, A. (1973). Structural coupling between pancreatic islet cells. Experientia 29, 1015-1018. doi: 10.1007/BF01930436

Palacios-Prado, N., Briggs, S. W., Skeberdis, V. A., Pranevicius, M., Bennett, M. V., and Bukauskas, F. F. (2010). pH-dependent modulation of voltage gating in connexin 45 homotypic and connexin $45 /$ connexin 43 heterotypic gap junctions. Proc. Natl. Acad Sci. U.S.A. 107, 9897-9902. doi: 10.1073/pnas.1004552107

Palacios-Prado, N., Chapuis, S., Panjkovich, A., Fregeac, J., Nagy, J. I., and Bukauskas, F. F. (2014). Molecular determinants of magnesium-dependent synaptic plasticity at electrical synapses formed by connexin36. Nat. Communic. 5:4667. doi: $10.1038 /$ ncomms5667

Palacios-Prado, N., Hoge, G., Marandykina, A., Rimkute, L., Chapuis, S., Paulauskas, N., et al. (2013). Intracellular Magnesium-Dependent Modulation of Gap Junction Channels Formed by Neuronal connexin36. J. Neurosci. 33, 4741-4753. doi: 10.1523/JNEUROSCI.2825-12.2013

Papazachariou, I. M., Martinez-Isla, A., Efthimiou, E., Williamson, R. C., and Girgis, S. I. (2000). Magnesium deficiency in patients with chronic pancreatitis identified by an intravenous loading test. Clin. Chim. Acta 302, 145-154. doi: 10.1016/s0009-8981(00)00363-6

Peracchia, C. (2004). Chemical gating of gap junction channels; roles of calcium, pH and calmodulin. Biochim. Biophys. Acta 1662, 61-80. doi: 10.1016/j. bbamem.2003.10.020

Pérez-Armendariz, E. M. (2013). Connexin 36, a key element in pancreatic beta cell function. Neuropharmacology 75, 557-566. doi: 10.1016/j.neuropharm.2013.08. 015

Pinto, B. I., Pupo, A., Garcia, I. E., Mena-Ulecia, K., Martinez, A. D., Latorre, R., et al. (2017). Calcium binding and voltage gating in Cx46 hemichannels. Sci. Rep. 7:15851. doi: 10.1038/s41598-017-15975-5

Purnick, P. E., Benjamin, D. C., Verselis, V. K., Bargiello, T. A., and Dowd, T. L. (2000a). Structure of the amino terminus of a gap junction protein. Arch. Biochem. Biophys. 381, 181-190. doi: 10.1006/abbi.2000.1989

Purnick, P. E., Oh, S., Abrams, C. K., Verselis, V. K., and Bargiello, T. A. (2000b). Reversal of the gating polarity of gap junctions by negative charge substitutions in the N-terminus of connexin 32. Biophys. J. 79, 2403-2415. doi: 10.1016/ S0006-3495(00)76485-X
Ramadass, S., Basu, S., and Srinivasan, A. (2015). SERUM magnesium levels as an indicator of status of Diabetes Mellitus type 2. Diabetes Metab. Syndr. 9, 42-45. doi: 10.1016/j.dsx.2014.04.024

Richard, G., Smith, L. E., Bailey, R. A., Itin, P., Hohl, D., Epstein, E. H. Jr., et al. (1998). Mutations in the human connexin gene GJB3 cause erythrokeratodermia variabilis. Nat. Gen. 20, 366-369. doi: 10.1038/3840

Rimkute, L., Kraujalis, T., Snipas, M., Palacios-Prado, N., Jotautis, V., Skeberdis, V. A., et al. (2018). Modulation of Connexin-36 Gap Junction Channels by Intracellular pH and Magnesium Ions. Front. Physiol. 9:362. doi: 10.3389/fphys. 2018.00362

Sanchez, H. A., and Verselis, V. K. (2014). Aberrant Cx26 hemichannels and keratitis-ichthyosis-deafness syndrome: insights into syndromic hearing loss. Front. Cell Neurosci. 8:354. doi: 10.3389/fncel.2014.00354

Sanchez, H. A., Villone, K., Srinivas, M., and Verselis, V. K. (2013). The D50N mutation and syndromic deafness: altered Cx26 hemichannel properties caused by effects on the pore and intersubunit interactions. J. Gen. Physiol. 142, 3-22. doi: 10.1085 /ggp.201310962

Senior, A. W., Evans, R., Jumper, J., Kirkpatrick, J., Sifre, L., Green, T., et al. (2020). Improved protein structure prediction using potentials from deep learning. Nature 577, 706-710. doi: 10.1038/s41586-019-1923-7

Serre-Beinier, V., Bosco, D., Zulianello, L., Charollais, A., Caille, D., Charpantier, E., et al. (2008). Cx36 makes channels coupling human pancreatic $\beta$-cells, and correlates with insulin expression. Human Mol. Gen. 18, 428-439. doi: 10.1093/ hmg/ddn 370

Siu, R. C. F., Kotova, A., Timonina, K., Zoidl, C., and Zoidl, G. R. (2021). Convergent NMDA receptor-Pannexin1 signaling pathways regulate the interaction of CaMKII with Connexin-36. Commun. Biol. 4:702. doi: 10.1038/ s42003-021-02230-x

Snipas, M., Kraujalis, T., Maciunas, K., Kraujaliene, L., Gudaitis, L., and Verselis, V. K. (2020). 4-state model for simulating kinetic and steady-state voltagedependent gating of gap junctions. Biophys. J. 119, 1640-1655. doi: 10.1016/j. bpj.2020.08.032

Srinivas, M., Verselis, V. K., and White, T. W. (2018). Human diseases associated with connexin mutations. Biochim. Biophys. Acta Biomembr. 1860, 192-201. doi: 10.1016/j.bbamem.2017.04.024

Symonds, M. R. E., and Moussalli, A. (2011). A brief guide to model selection, multimodel inference and model averaging in behavioural ecology using Akaike's information criterion. Behav. Ecol. Sociobiol. 65, 13-21. doi: 10.1007/ s00265-010-1037-6

Szoboszlay, M., Lorincz, A., Lanore, F., Vervaeke, K., Silver, R. A., and Nusser, Z. (2016). Functional Properties of Dendritic Gap Junctions in Cerebellar Golgi Cells. Neuron 90, 1043-1056. doi: 10.1016/j.neuron.2016.03.029

Tejada, M. G., Sudhakar, S., Kim, N. K., Aoyama, H., Shilton, B. H., and Bai, D. (2018). Variants with increased negative electrostatic potential in the Cx50 gap junction pore increased unitary channel conductance and magnesium modulation. Biochem. J. 475, 3315-3330. doi: 10.1042/BCJ20180523

Tong, J. J., Khan, U., Haddad, B. G., Minogue, P. J., Beyer, E. C., Berthoud, V. M., et al. (2021). Molecular mechanisms underlying enhanced hemichannel function of a cataract-associated Cx50 mutant. Biophys. J. 120, 5644-5656. doi: $10.1016 /$ j.bpj.2021.11.004

Trexler, E. B., Bukauskas, F. F., Bennett, M. V. L., Bargiello, T. A., and Verselis, V. K. (1999). Rapid and direct effects of $\mathrm{pH}$ on connexins revealed by the connexin 46 hemichannel preparation. J. Gen. Physiol. 113, 721-742. doi: 10.1085/jgp.113.5. 721

Verselis, V. K., Ginter, C. S., and Bargiello, T. A. (1994). Opposite voltage gating polarities of two closely related connexins. Nature 368, 348-351. doi: 10.1038/ $368348 \mathrm{a} 0$

Vink, R., Cook, N. L., and Van Den Heuvel, C. (2009). Magnesium in acute and chronic brain injury: an update. Magnes. Res. 22, 158s-162s. doi: 10.1684/mrh. 2009.0175

Wang, H. Y., Lin, Y. P., Mitchell, C. K., Ram, S., and O'brien, J. (2015). Twocolor fluorescent analysis of connexin 36 turnover: relationship to functional plasticity. J. Cell Sci. 128, 3888-3897. doi: 10.1242/jcs.162586

White, T. W., Wang, H., Mui, R., Litteral, J., and Brink, P. R. (2004). Cloning and functional expression of invertebrate connexins from Halocynthia pyriformis. FEBS Lett. 577, 42-48. doi: 10.1016/j.febslet.2004.09.071

Wu, E. L., Cheng, X., Jo, S., Rui, H., Song, K. C., Dávila-Contreras, E. M., et al. (2014). CHARMM-GUI membrane builder toward realistic biological 
membrane simulations. J. Comput. Chem. 35, 1997-2004. doi: 10.1002/jcc. 23702

Yifrach, O. (2004). Hill coefficient for estimating the magnitude of cooperativity in gating transitions of voltage-dependent ion channels. Biophys. J. 87, 822-830. doi: 10.1529/biophysj.104.040410

Yue, B., Haddad, B. G., Khan, U., Chen, H., Atalla, M., Zhang, Z., et al. (2021). Connexin 46 and connexin 50 gap junction channel properties are shaped by structural and dynamic features of their N-terminal domains. J. Physiol. 599, 3313-3335. doi: 10.1113/JP281339

Conflict of Interest: The authors declare that the research was conducted in the absence of any commercial or financial relationships that could be construed as a potential conflict of interest.
Publisher's Note: All claims expressed in this article are solely those of the authors and do not necessarily represent those of their affiliated organizations, or those of the publisher, the editors and the reviewers. Any product that may be evaluated in this article, or claim that may be made by its manufacturer, is not guaranteed or endorsed by the publisher.

Copyright (c) 2022 Kraujalis, Gudaitis, Kraujaliene, Snipas, Palacios-Prado and Verselis. This is an open-access article distributed under the terms of the Creative Commons Attribution License (CC BY). The use, distribution or reproduction in other forums is permitted, provided the original author(s) and the copyright owner(s) are credited and that the original publication in this journal is cited, in accordance with accepted academic practice. No use, distribution or reproduction is permitted which does not comply with these terms. 